\title{
Synthesis of novel $\beta$-lactams and in vitro evaluation against the human malaria parasite Plasmodium falciparum
}

\author{
Margaret A. L. Blackie, ${ }^{\mathrm{a}}$ Tzu-Shean Feng, ${ }^{\mathrm{b}}$ Peter J. Smith, ${ }^{\mathrm{c}}$ and Kelly Chibale ${ }^{\mathrm{b}, \mathrm{d}}$ \\ ${ }^{a}$ Department of Chemistry and Polymer Science, Stellenbosch University, Private Bag XI \\ Matieland, South Africa \\ ${ }^{b}$ Department of Chemistry, University of Cape Town, Cape Town, South Africa \\ ${ }^{c}$ Department of Pharmacology, University of Cape Town, Cape Town, South Africa \\ ${ }^{d}$ Institute of Drug Discovery and Molecular Medicine, University of Cape Town, Cape Town, \\ South Africa \\ E-mail:mblackie@sun.ac.za
}

DOI: $\underline{\text { http://dx.doi.org/10.3998/ark.5550190.p009.465 }}$

\begin{abstract}
The use of three-component four-center Ugi reactions to afford the synthesis of novel $\beta$-lactams is described. Three series of compounds have been made using this reaction in combination with the Mannich reaction. All compounds formed are racemic mixtures and were tested against the chloroquine sensitive D10 strain of Plasmodium falciparum in an in vitro assay. All compounds showed low to moderate microMolar range activity with the most active compounds 2.6 and 3.1 showing $\mathrm{IC}_{50}$ values $16 \mu \mathrm{M}$ and $15 \mu \mathrm{M}$ respectively
\end{abstract}

Keywords: Ugi reaction, Mannich reaction, $\beta$-lactam, antiplasmodial

\section{Introduction}

Malaria continues to be a major blight with almost half the world's population at risk. ${ }^{1}$ Whilst the disease is currently primarily endemic in Third World countries, the socio-economic impact of this disease is felt worldwide. Each year an approximated 5\% of the world's population succumbs to infection by parasites of the genus Plasmodium. The level of drug resistance continues to rise. Most recently the emergence of resistance to artemesinin based therapies is of grave concern. ${ }^{2}$ The result is an urgent need for new pharmacophores to bypass these resistance mechanisms. Modern medicinal chemistry research is driven by the need to identify, develop and optimise lead compounds in the most cost-effective and efficient way possible. The use of multicomponent reactions (MCRs) which utilise at least three different starting materials in a 
one-pot synthesis is an important synthetic strategy in this environment. ${ }^{3}$ MCRs give rise to a wide variety of structural diversity via simple methodology. ${ }^{4}$

Ever since the structure of penicillin was elucidated, the $\beta$-lactam moiety has been of interest. ${ }^{5}$ In recent years there has been an increasing focus on $\beta$-lactams and their application to a wide variety of infectious diseases. ${ }^{6}$ The exploration of the application of $\beta$-lactams in the treatment of malaria is an area of serious research interest. The fact that $\beta$-lactams are easily accessed via a three-component four-centre (3C-4CR) Ugi type reaction makes this class of compounds attractive. ${ }^{4}$

We have therefore synthesised a series of $\beta$-lactams, using a 3C-4CR protocol, from commercially available starting materials in order to lay a foundation of structure-activity information which will allow a more focused second phase of synthesis and biological evaluation. We report herein, the synthesis and antiplasmodial activity of these $\beta$-lactams.

Three series of novel $\beta$-lactams have been synthesised and general structures are shown in Figure 1 . Series $1: R^{2}$ was a cyclohexyl group and $R^{1}$ was varied. In most cases $R^{1}$ was a substituted phenyl ring, but there are some exceptions. Series 2 : $\mathrm{R}^{1}$ was kept constant and the starting isocyanide was varied. Series $3: R^{1}$ was varied at the terminal tertiary amine and $R^{2}$ was again a cyclohexyl group. The basic backbone formed from the Ugi reaction was preserved.<smiles>[R]NC(=O)C([R])N1CCC1=O</smiles>

where $\quad \mathrm{R}^{1} \quad \mathrm{R}^{2}$

Series $1 \quad$ various

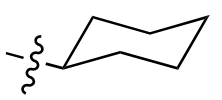

Series 2

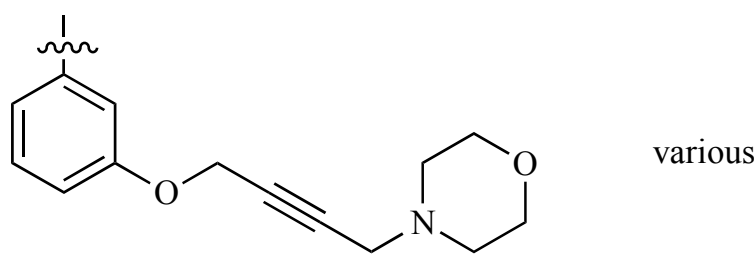

Series 3



Figure 1. General structure of the three series of novel $\beta$-lactams synthesised. 


\section{Results and Discussion}

Series 1 was synthesized from $\beta$-alanine, cyclohexyl isocyanide and the chosen aldehyde, Scheme 1. Reactions were carried out in methanol at $25^{\circ} \mathrm{C}$. The reaction time varied from $48-72$ hours and good yields were obtained. Reactions were carried out in a small volume of solvent in order to maximize yields and minimize reaction times. The use of low molecular weight alcohols or aprotic polar solvents has been shown to be advantageous in Ugi methodology hence the choice of methanol as the solvent. ${ }^{7}$ Pirrung and Das Sarma noted that biphasic systems using aqueous media accelerate this type of reactions. ${ }^{8}$ This was attempted, but we found that the convenience of removal of the methanol and immediate addition to the silica gel column for purification outweighed any advantage to the use of a biphasic system.

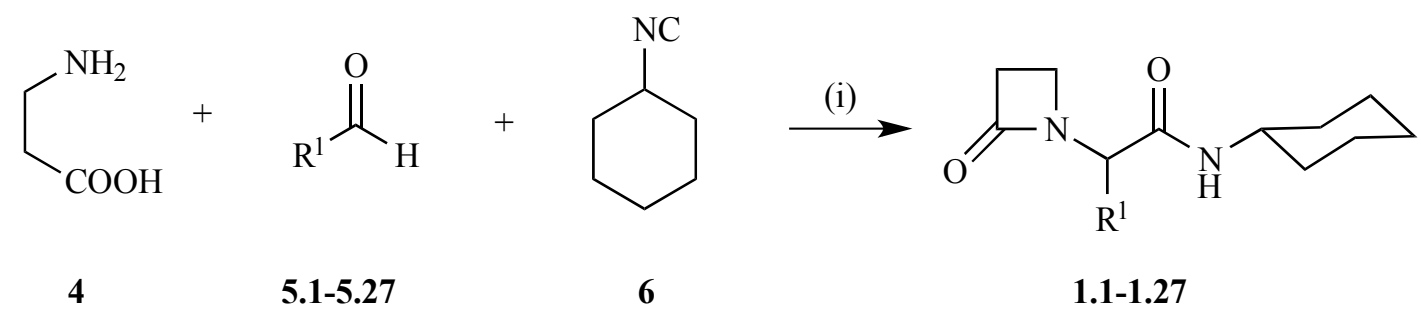

Scheme 1. Synthesis of series 1 via the Ugi reaction (i) methanol, $25^{\circ} \mathrm{C}, 48-72 \mathrm{~h}$.

Series 2 and 3 (compounds 2.1 - 2.7 and 3.1 $-\mathbf{3 . 8}$ respectively) were synthesised from 3(prop-2-ynyloxy)benzaldehyde 5.15. This was prepared from 3-hydroxybenzaldehyde, 7, and propargyl bromide 6 following the procedure described by Pitlik and Townsend. ${ }^{9}$ Propargyl bromide was added dropwise to a mixture of the benzaldehyde in anhydrous DMF at $25{ }^{\circ} \mathrm{C}$ and potassium carbonate was used as the base. The reaction was stirred for 18 hours and gave the product in excellent yield.

In series $\mathbf{2}$, compounds $\mathbf{2 . 1} \mathbf{- 2 . 7}$, cyclohexyl isocyanide was used throughout the series, so the Ugi reaction to form the $\beta$-lactam was performed first using the same conditions described for series 1. Thus $\beta$-alanine 4, cyclohexyl isocyanide 6 and 3-(prop-2-ynyloxy)benzaldehyde $\mathbf{5 . 1 5}$ were mixed together in methanol at room temperature and allowed to stir for $48-72$ hours. Compound $\mathbf{1 . 1 5}$ was isolated as a white crystalline solid in $81 \%$ yield after purification by column chromatography.

Compounds 1.15 was then used as a reagent in the Mannich reaction. The terminal acetylene 1.15 was reacted with aqueous formaldehyde, and a series of different amines (1.2 eq) in the presence of a catalytic amount of copper(I) iodide in DMSO. The reaction was allowed to stir at $25^{\circ} \mathrm{C}$ for 2 hours before the reaction was quenched with aqueous base. Compounds 2.1 - 2.7 were isolated in good to excellent yields. 
<smiles>O=Cc1cccc(O)c1</smiles>

7<smiles>CC#CCBr</smiles>

8

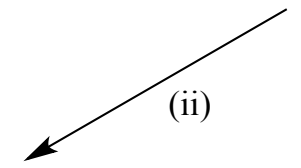<smiles>C#CCOc1cccc(C=O)c1</smiles>

5.15<smiles>C#CCOc1cccc(C(C(=O)NC2CCCCC2)N2CCC2=O)c1</smiles>

1.15 (iii) $\checkmark$<smiles>[R]N([R])CC#CCOc1cccc(C(C(=O)NC2CCCC2)N2CCC2=O)c1</smiles>

$2.1-2.7$

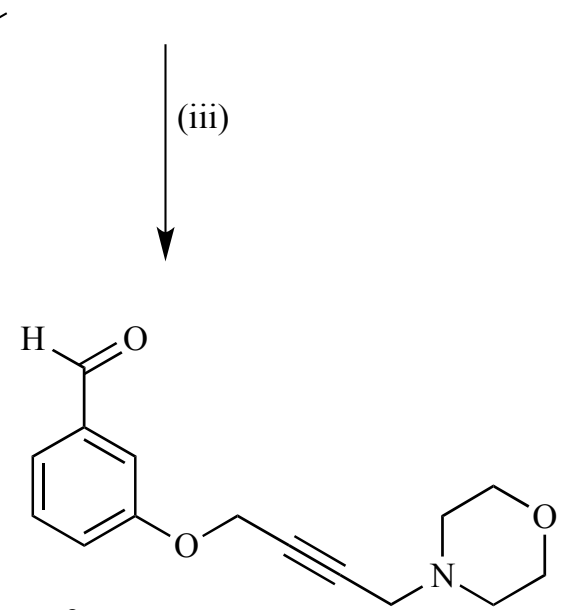

9<smiles>[R]NC(=O)C(c1cccc(OCC#CCN2CCOCC2)c1)N1CCC1=O</smiles>

$3.1-3.8$

Scheme 2. Synthesis of series 2 and 3 via Ugi and Mannich reactions (i) $\mathrm{K}_{2} \mathrm{CO}_{3}$, anhydrous DMF, $25^{\circ} \mathrm{C}, 18 \mathrm{~h}$; (ii) methanol, $25^{\circ} \mathrm{C}, 48-72 \mathrm{~h}$; (iii) aqueous formaldehyde, $\mathrm{Cu}$ (I) iodide, DMSO, $25^{\circ} \mathrm{C}, 2 \mathrm{~h}$.

The synthetic strategy was reversed in pursuit of series 3 where the Mannich reaction was performed using 3-(prop-2-ynyloxy)benzaldehyde 5.15, morpholine and aqueous formaldehyde to form compound 9. Compound 9 was then reacted with $\beta$-alanine and a series of isocyanides to give compounds $3.1-\mathbf{3 . 9}$ in good to excellent yields.

All compounds in series 1, 2 and $\mathbf{3}$ were isolated as racemic mixtures. The chiral carbon is adjacent to the $\beta$-lactam ring. The final products were all characterized fully. Several definitive elements of characterization were identified. As a result of the presence of chiral carbon atom, in 
the ${ }^{1} \mathrm{H}$ NMR spectra, the four protons on the $\beta$-lactam ring give rise to two doublet of triplets (at approximately 3.6 and $3.1 \mathrm{ppm}$ ) and two doublet of doublet of doublets (at approximately 2.9 and $2.8 \mathrm{ppm}$ ). These signals act as a fingerprint for the desired product. The presence of the carbonyl carbons is clear in both the ${ }^{13} \mathrm{C}$ NMR spectra and in the IR spectra lending further support to the formation of the desired product. All characterisation data is consistent with the given structures proposed including high resolution mass spectrometry and elemental analysis. No attempt was made to separate the two enantiomers and all testing and characterisation was carried out on the racemic mixtures.

A number of the newly synthesised $\beta$-lactams showed moderate activity against the chloroquine sensitive D10 of P. falciparum, Table 1. The compounds in series $\mathbf{2}$ and $\mathbf{3}$ were generally more active than the compounds of series 1 .

Table 1. Results of in vitro testing against CQ sensitive D10 of $P$. falciparum

\begin{tabular}{|c|c|c|c|}
\hline $\mathrm{Cpd} \mathrm{No}{ }^{\mathrm{a}}$ & $\mathrm{R}^{1}$ & $\mathrm{R}^{2}$ & $\begin{array}{c}\mathrm{IC}_{50} \mu \mathrm{M} \\
\mathrm{D} 10\end{array}$ \\
\hline CQ & - & _ & 0.032 \\
\hline 1.1 & & & 281 \\
\hline 1.2 & & & 35 \\
\hline 1.3 & & & 58 \\
\hline 1.4 & & & 187 \\
\hline 1.5 & & & 80 \\
\hline 1.6 & & & $>331$ \\
\hline 1.7 & & & $>316$ \\
\hline 1.8 & & & 246 \\
\hline 1.9 & & & $>294$ \\
\hline 1.10 & & & $>274$ \\
\hline 1.11 & & & 251 \\
\hline
\end{tabular}


Table 1. Continues

1.12


Table 1. Continues

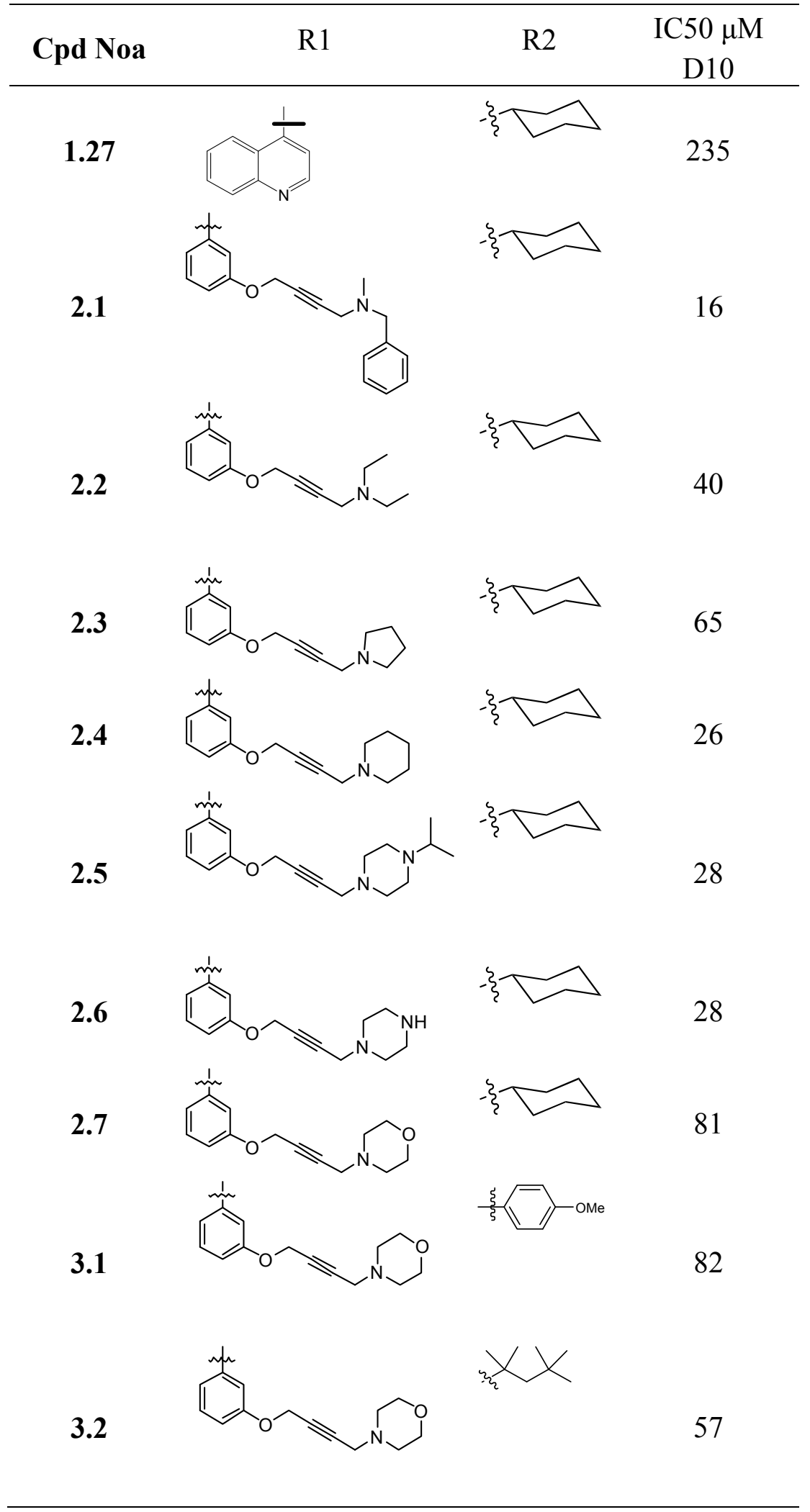


Table 1. Continues

Cpd Noa

\section{Conclusions}

In series $\mathbf{1}$, compounds $1.19\left(\mathrm{IC}_{50} 27 \mu \mathrm{M}\right), \mathbf{1 . 2}\left(\mathrm{IC}_{50} 35 \mu \mathrm{M}\right)$ and $\mathbf{1 . 1 5}\left(\mathrm{IC}_{50} 48 \mu \mathrm{M}\right)$ were the most efficacious. The data shows that, in general, for series $\mathbf{1}$, the presence of an electronwithdrawing group on the aromatic group results in compounds that are more active than those which have an electron-donating group. Clearly the pattern of activity is complex, but it is generally true that those compounds with an electron-withdrawing group in the para position are more active than those bearing electron-withdrawing groups at other positions. When there are several substituents on the aromatic ring no clear pattern is evident. It was also evident that hydroxyl and methoxy substitution had a detrimental impact on efficacy regardless of position on the phenyl ring.

Compound $\mathbf{1 . 1 5}$ which had shown relatively good efficacy against the D10 strain of $P$. falciparum was selected as the basis for the series $\mathbf{2}$ and 3. In series $\mathbf{2}$ there is a smaller variation in efficacy relative to series $\mathbf{1}$. The nature of the terminal amine group is significant. The 
methylbenzyl terminal amine present in $\mathbf{2 . 1}\left(\mathrm{IC}_{50} 15.7 \mu \mathrm{M}\right)$ shows greatest efficacy, whilst the morpholine group shows least efficacy. In series $\mathbf{3}$, the variation of $\mathrm{R}^{2}$ had some observable impact on the efficacy against the D10 strain. The most active was the benzotriazole compound, 3.7 and the least efficacious was the compound bearing the monobranched alkyl side-chain, 3.3.

The addition of a terminal amine group appears to have the overall effect of improving efficacy. However, the efficacy achieved by this class of compounds is moderate at best. Once the target of these molecules has been elucidated, further modification using rational design may prove useful.

\section{Experimental Section}

General. All chemicals were used as supplied by Aldrich. ${ }^{1} \mathrm{H}$ and ${ }^{13} \mathrm{C}$ spectra were recorded at room temperature on Varian EM 400 or $300 \mathrm{MHz}$ spectrometers. Infrared spectra were recorded on a Perkin-Elmer Paragon 1000 FT IR spectrometer. Melting points were performed on a Kofler hot stage microscope (Reichert Thermovar). Elemental analyses were performed using a Carlo Erba EA1108 elemental analyser in the microanalytical laboratory of the University of Cape Town.

Compounds were tested in duplicate on one occasion against chloroquine sensitive (CQS) strain of Plasmodium falciparum (D10). Continuous in vitro cultures of asexual erythrocyte stages of $P$. falciparum were maintained using the modified method of Trager and Jensen. ${ }^{10}$ Quantitative assessment of antiplasmodial activity in vitro was determined via the parasite lactate dehydrogenase assay using the modified method described by Makler. ${ }^{11}$ The samples were prepared to a $2 \mathrm{mg} / \mathrm{mL}$ stock solution in $10 \% \mathrm{DMSO}$ or $10 \%$ methanol and were sonicated to enhance solubility. Samples were tested as a suspension if not completely dissolved. Stock solutions were stored at $-20{ }^{\circ} \mathrm{C}$. Further dilutions were prepared on the day of the experiment. Chloroquine (CQ) was used as the reference drug in all experiments. A full dose-response was performed for all compounds to determine the concentration inhibiting 50\% of parasite growth $\left(\mathrm{IC}_{50}\right.$ - value). Test samples were tested at a starting concentration of $100 \mu \mathrm{g} / \mathrm{mL}$, which was then serially diluted two-fold in complete medium to give 10 concentrations; with the lowest concentration being $0.2 \mu \mathrm{g} / \mathrm{mL}$. The same dilution technique was used for all samples. CQ was tested at a starting concentration of $100 \mathrm{ng} / \mathrm{mL}$. The highest concentration of solvent to which the parasites were exposed had no measurable effect on the parasite viability (data not shown). The $\mathrm{IC}_{50}$-values were obtained using a non-linear dose-response curve fitting analysis via Graph Pad Prism v.4.0 software.

3-(Prop-2-ynyloxy)benzaldehyde (5.15). Propargyl bromide $(3.2 \mathrm{~mL}, 80 \% \mathrm{w} / \mathrm{w}$ solution in toluene, $28.9 \mathrm{mmol})$ was added dropwise to a mixture of 3-hydroxybenzaldehyde $(3.0 \mathrm{~g}, 24.1$ $\mathrm{mmol})$ and $\mathrm{K}_{2} \mathrm{CO}_{3}(4.99 \mathrm{~g}, 36.1 \mathrm{mmol})$ in anhydrous DMF $(30 \mathrm{~mL})$ at rt, and stirred for $18 \mathrm{~h}$. The reaction mixture was then diluted with $\mathrm{H}_{2} \mathrm{O}(100 \mathrm{~mL})$, and extracted with EtOAc $(4 \mathrm{x} 50$ 
$\mathrm{mL})$, washed with $\mathrm{H}_{2} \mathrm{O}(4 \times 200 \mathrm{~mL})$ and brine $(150 \mathrm{~mL})$, dried $\left(\mathrm{Na}_{2} \mathrm{SO}_{4}\right)$ and solvent removed to yield the crude product. The crude product was purified via column chromatography, eluting with EtOAc/hexane (1:9) to yield the pure product as a yellow oil $(3.54 \mathrm{~g}, 93 \%) ;^{12} \mathrm{R}_{\mathrm{f}}$ (EtOAc/hexane, 2:8) 0.48. ${ }^{1} \mathrm{H}$ NMR $\left(\mathrm{CDCl}_{3}, 400 \mathrm{MHz}, 30{ }^{\circ} \mathrm{C}\right): \delta_{\mathrm{H}} 9.97(\mathrm{~s}, 1 \mathrm{H}), 7.49(\mathrm{~m}, 3 \mathrm{H})$, $7.25(\mathrm{~m}, 1 \mathrm{H}), 4.75(\mathrm{~d}, J 2.4 \mathrm{~Hz}, 2 \mathrm{H}), 2.54(\mathrm{t}, J 2.4 \mathrm{~Hz}, 1 \mathrm{H})$.

General synthesis for $\boldsymbol{\beta}$-lactams. $\beta$-Alanine (1.1 equiv), cyclohexyl isocyanide (1.0 equiv) and the appropriate aldehyde (1.0 equiv) were added to $\mathrm{MeOH}$ and allowed to stir at $25{ }^{\circ} \mathrm{C}$ for $48-72$ h. The reaction was monitored by TLC (1:1 EtOAc/hexane). Once the aldehyde spot had disappeared the reaction was deemed complete. The solvent was removed from the reaction mixture under reduced pressure. The crude mixture was purified using silica gel chromatography eluting with 1:1 EtOAc/hexane. This method is a slight variation on the method described by Pitlik and Townsend. ${ }^{9}$

N-Cyclohexyl-2-(4-fluorophenyl)-2-(2-oxoazetidin-1-yl)acetamide (1.1). White crystalline solid; Yield 74\%; mp 126-127 ${ }^{\circ} \mathrm{C}$ (EtOAc/hexane); IR (KBr, $\left.v_{\max }, \mathrm{cm}^{-1}\right) 3276 \mathrm{~s}, 2931 \mathrm{~s}, 2855 \mathrm{~m}$, 1739vs, 1651vs, 1510s, 1225s, 1161m, 808m; ${ }^{1} \mathrm{H}$ NMR $\left(\mathrm{CDCl}_{3}, 300 \mathrm{MHz}, 30{ }^{\circ} \mathrm{C}\right): \delta_{\mathrm{H}} 7.34(\mathrm{~m}$, $2 \mathrm{H}), 7.05(\mathrm{~m}, 2 \mathrm{H}), 6.25(\mathrm{~d}, J 7.5 \mathrm{~Hz}, 1 \mathrm{H}), 5.33(\mathrm{~s}, 1 \mathrm{H}), 3.76(\mathrm{~m}, 1 \mathrm{H}), 3.60(\mathrm{td}, J 5.6,5.6,2.8 \mathrm{~Hz}$, $1 \mathrm{H}), 3.16(\mathrm{td}, J 5.6,5.6,2.8 \mathrm{~Hz}, 1 \mathrm{H}), 2.98(\mathrm{ddd}, J 14.8,5.5,2.8 \mathrm{~Hz}, 1 \mathrm{H}) 2.85$ (ddd, $J 14.8,5.5$, $2.8 \mathrm{~Hz}, 1 \mathrm{H}) ; 1.86(\mathrm{~m}, 2 \mathrm{H}), 1.62(\mathrm{~m}, 3 \mathrm{H}), 1.32(\mathrm{~m}, 2 \mathrm{H}), 1.12(\mathrm{~m}, 3 \mathrm{H}) ;{ }^{13} \mathrm{C} \mathrm{NMR}\left(\mathrm{CDCl}_{3}, 100\right.$ $\mathrm{MHz}, 30{ }^{\circ} \mathrm{C}$ ): $\delta_{\mathrm{c}} 167.9$ (IV), 167.5 (IV), 164.0 (IV), 161.5 (IV), 130.9 (IV), 130.9, 130.0, 116.1, 115.9, 50.3, 48.5, 39.0, 36.2, 32.7, 25.5, 24.7, 24.7; HRMS $m / z 304.1587\left[\mathrm{M}^{+}, \mathrm{C}_{17} \mathrm{H}_{21} \mathrm{FN}_{2} \mathrm{O}_{2}\right.$ requires 304.1587]; Anal. Calcd for $\mathrm{C}_{17} \mathrm{H}_{21} \mathrm{FN}_{2} \mathrm{O}_{2}$ : C, 67.09; H, 6.95; N, 9.20. Found: C, 66.72; $\mathrm{H}, 6.59 ; \mathrm{N}, 9.30$.

2-(4-Chlorophenyl)-N-cyclohexyl-2-(2-oxoazetidin-1-yl)acetamide (1.2). White crystalline solid; Yield 77\%; mp 127-129 ${ }^{\circ} \mathrm{C}$ (EtOAc/hexane); IR (KBr, $\left.v_{\max }, \mathrm{cm}^{-1}\right) 3285 \mathrm{~m}, 2933 \mathrm{~m}, 1739 \mathrm{vs}$, $1651 \mathrm{vs}, 1539 \mathrm{~m}, 1429 \mathrm{~m}, 1257 \mathrm{~m}, 1091 \mathrm{~m} ;{ }^{1} \mathrm{H} \mathrm{NMR}\left(\mathrm{CDCl}_{3}, 400 \mathrm{MHz}, 30{ }^{\circ} \mathrm{C}\right): \delta_{\mathrm{н}} 7.38(\mathrm{~d}, J$ $8.4 \mathrm{~Hz}, 2 \mathrm{H}), 7.33(\mathrm{~d}, J 8.4 \mathrm{~Hz}, 2 \mathrm{H}), 6.06(\mathrm{~d}, J 7.7 \mathrm{~Hz}, 1 \mathrm{H}), 5.26(\mathrm{~s}, 1 \mathrm{H}), 3.82(\mathrm{~m}, 1 \mathrm{H}), 3.61(\mathrm{td}, J$ $5.5,5.5,2.6 \mathrm{~Hz}, 1 \mathrm{H}), 3.20(\mathrm{td}, J 5.5,5.5,2.6 \mathrm{~Hz}, 1 \mathrm{H}), 3.06(\mathrm{ddd}, J 14.8,5.5,2.6 \mathrm{~Hz}, 1 \mathrm{H}), 2.93$ $(\mathrm{ddd}, J 14.8,5.5,2.6 \mathrm{~Hz}, 1 \mathrm{H}) ; 1.93(\mathrm{~m}, 2 \mathrm{H}), 1.74(\mathrm{~m}, 3 \mathrm{H}), 1.42(\mathrm{~m}, 2 \mathrm{H}), 1.22(\mathrm{~m}, 3 \mathrm{H}) ;{ }^{13} \mathrm{C}$ NMR $\left(\mathrm{CDCl}_{3}, 100 \mathrm{MHz}, 30{ }^{\circ} \mathrm{C}\right): \delta_{\mathrm{c}} 167.9$ (IV), 167.2 (IV), 137.8, 134.7, 133.5, 129.5, 129.2, 70.7, 59.6, 48.7, 39.0, 36.0, 32.8, 25.4, 24.7, 24.7; HRMS m/z 320.1290 [ $\mathrm{M}^{+}, \mathrm{C}_{17} \mathrm{H}_{21} \mathrm{ClN}_{2} \mathrm{O}_{2}$ requires 320.1292]; Anal. Calcd for $\mathrm{C}_{17} \mathrm{H}_{21} \mathrm{ClN}_{2} \mathrm{O}_{2}$ : C, 63.64; H, 6.60; N, 8.73. Found: C, 63.30; H, 6.53; N, 8.67.

2-(4-Bromophenyl)- $\boldsymbol{N}$-cyclohexyl-2-(2-oxoazetidin-1-yl)acetamide (1.3). White crystalline solid, Yield: 84\%; mp 94-95 ${ }^{\circ} \mathrm{C}$ (EtOAc/hexane); IR (KBr, $\left.v_{\max }, \mathrm{cm}^{-1}\right) 3304 \mathrm{~m}, 2931 \mathrm{~s}, 2854 \mathrm{~m}$, $1741 \mathrm{vs}, 1657 \mathrm{vs}, 1644 \mathrm{~m}, 1488 \mathrm{~m}, 1012 \mathrm{~m} ;{ }^{1} \mathrm{H} \mathrm{NMR}\left(\mathrm{CDCl}_{3}, 400 \mathrm{MHz}, 30{ }^{\circ} \mathrm{C}\right): \delta_{\mathrm{H}} 7.52(\mathrm{~d}, J$ $8.8 \mathrm{~Hz}, 2 \mathrm{H}), 7.26(\mathrm{~d}, J 8.8 \mathrm{~Hz}, 2 \mathrm{H}), 6.33(\mathrm{br} \mathrm{d}, J 7.7 \mathrm{~Hz}, 1 \mathrm{H}), 5.32(\mathrm{~s}, 1 \mathrm{H}), 3.80(\mathrm{~m}, 1 \mathrm{H}), 3.628(\mathrm{td}$, $J 5.8,5.8,2.7 \mathrm{~Hz}, 1 \mathrm{H}), 3.20(\mathrm{td}, J 5.8,5.8,2.7 \mathrm{~Hz}, 1 \mathrm{H}), 3.02$ (ddd, $J 14.8,5.8,2.7 \mathrm{~Hz}, 1 \mathrm{H}), 2.90$ $(\mathrm{ddd}, J 14.8,5.8,2.7 \mathrm{~Hz}, 1 \mathrm{H}), 1.90(\mathrm{~m}, 2 \mathrm{H}), 1.71(\mathrm{~m}, 3 \mathrm{H}), 1.40(\mathrm{~m}, 2 \mathrm{H}), 1.19(\mathrm{~m}, 3 \mathrm{H}) ;{ }^{13} \mathrm{C} \mathrm{NMR}$ $\left(\mathrm{CDCl}_{3}, 100 \mathrm{MHz}, 3{ }^{\circ} \mathrm{C}\right): \delta_{\mathrm{c}} 167.9$ (IV), 167.2 (IV), 134.0, 132.2, 129.8, 122.8, 95.0, 70.7, 48.7, 
39.7, 36.3, 32.7, 25.4, 24.7, 24.6; HRMS m/z 364.0785 [ $\mathrm{M}^{+}, \mathrm{C}_{17} \mathrm{H}_{21} \mathrm{BrN}_{2} \mathrm{O}_{2}$ requires 364.0786]; Anal. Calcd for $\mathrm{C}_{17} \mathrm{H}_{21} \mathrm{BrN}_{2} \mathrm{O}_{2}$ : C, 55.90; H, 5.79; N, 7.67. Found: C, 55.70; H, 5.80; N, 7.56.

$\mathrm{N}$-Cyclohexyl-2-(4-iodophenyl)-2-(2-oxoazetidin-1-yl)acetamide (1.4). White crystalline solid, Yield: $84 \%$; mp 127-129 ${ }^{\circ} \mathrm{C}$ (EtOAc/hexane); IR (KBr, $\left.v_{\max }, \mathrm{cm}^{-1}\right) 3288 \mathrm{~s}, 2931 \mathrm{~s}, 1743 \mathrm{vs}, 1651 \mathrm{vs}$, $1561 \mathrm{~s}, 1486 \mathrm{~m}, 1249 \mathrm{~m}, 1008 \mathrm{~m} ;{ }^{1} \mathrm{H} \mathrm{NMR}\left(\mathrm{CDCl}_{3}, 400 \mathrm{MHz}, 30{ }^{\circ} \mathrm{C}\right): \delta_{\mathrm{H}} 7.69(\mathrm{~d}, J 8.4 \mathrm{~Hz}, 2 \mathrm{H})$, $7.09(\mathrm{~d}, J 8.4 \mathrm{~Hz}, 2 \mathrm{H}), 6.07(\mathrm{~d}, J 8.0 \mathrm{~Hz}, 1 \mathrm{H}), 5.20(\mathrm{~s}, 1 \mathrm{H}), 3.78(\mathrm{~m}, 1 \mathrm{H}), 3.57$ (td, $J 5.5,5.5$, $2.6 \mathrm{~Hz}, 1 \mathrm{H}) 3.16(\mathrm{td}, J 5.5,5.5,2.6 \mathrm{~Hz}, 1 \mathrm{H}), 3.00$ (ddd, $J 14.6,5.5,2.6 \mathrm{~Hz}, 1 \mathrm{H}), 2.88$ (ddd, $J$ 14.6, $5.5,2.6 \mathrm{~Hz}, 1 \mathrm{H}), 1.88(\mathrm{~m}, 2 \mathrm{H}), 1.69(\mathrm{~m}, 3 \mathrm{H}), 1.37(\mathrm{~m}, 2 \mathrm{H}), 1.19(\mathrm{~m}, 3 \mathrm{H}) ;{ }^{13} \mathrm{C} \mathrm{NMR}\left(\mathrm{CDCl}_{3}, 100\right.$ $\mathrm{MHz}, 30{ }^{\circ} \mathrm{C}$ ): $\delta_{\mathrm{c}} 167.8$ (IV), 167.1 (IV), 138.0 (2C), 134.7 (IV), 129.8 (2C), 94.2 (IV), 58.9, 48.5, 39.1, 36.2, 32.5, 25.3, 24.6, 24.5; HRMS m/z 412.0469 [M $\mathrm{M}^{+}, \mathrm{C}_{17} \mathrm{H}_{21} \mathrm{IN}_{2} \mathrm{O}_{2}$ requires 412.0468]; Anal. Calcd for $\mathrm{C}_{17} \mathrm{H}_{21} \mathrm{IN}_{2} \mathrm{O}_{2}$ : C, 49.53; H, 5.13; N, 6.79. Found: C, 49.52; H, 5.14; $\mathrm{N}, 6.47$.

$\mathrm{N}$-Cyclohexyl-2-(2-oxoazetidin-1-yl)-2-(4-(trifluoromethyl)phenyl)acetamide (1.5). Yellow crystalline solid; Yield 64\%; mp 115-116 ${ }^{\circ} \mathrm{C}$ (EtOAc/hexane); IR (KBr, $\left.v_{\max }, \mathrm{cm}^{-1}\right) 3278 \mathrm{~s}, 2930 \mathrm{~s}$, 2856m, 1746vs, 1646vs, 1330vs, 1127vs, 703m; ${ }^{1} \mathrm{H} \mathrm{NMR}\left(\mathrm{CDCl}_{3}, 400 \mathrm{MHz}, 30{ }^{\circ} \mathrm{C}\right): \delta_{\mathrm{H}} 7.61(\mathrm{~m}$, $4 \mathrm{H}), 6.42$ (br d, $J 6.4 \mathrm{~Hz}, 1 \mathrm{H}), 5.43(\mathrm{~s}, 1 \mathrm{H}), 3.79(\mathrm{~m}, 1 \mathrm{H}), 3.64(\mathrm{td}, J 5.5,5.5,2.9 \mathrm{~Hz}, 1 \mathrm{H}), 3.21$ $(\mathrm{td}, J 5.5,5.5,2.9 \mathrm{~Hz}, 1 \mathrm{H}), 3.03(\mathrm{ddd}, J 15.0,5.5,2.7 \mathrm{~Hz}, 1 \mathrm{H}), 2.91$ (ddd, $J 15.0,5.5,2.7 \mathrm{~Hz}, 1 \mathrm{H})$, $1.88(\mathrm{~m}, 2 \mathrm{H}), 1.62(\mathrm{~m}, 3 \mathrm{H}), 1.37(\mathrm{~m}, 2 \mathrm{H}), 1.18(\mathrm{~m}, 3 \mathrm{H}) ;{ }^{13} \mathrm{C} \mathrm{NMR}\left(\mathrm{CDCl}_{3}, 100 \mathrm{MHz}, 30{ }^{\circ} \mathrm{C}\right): \delta_{\mathrm{c}}$ 168.0 (IV), 166.9 (IV), 136.1 (IV), 131.4, 129.5, 125.4, 125.4, 124.9, 59.6, 48.8, 39.2, 36.4, 32.7, 32.7, 25.4, 24.7, 24.6; HRMS m/z 354.1557 [ $\mathrm{M}^{+}, \mathrm{C}_{18} \mathrm{H}_{21} \mathrm{~F}_{3} \mathrm{~N}_{2} \mathrm{O}_{2}$ requires 354.1555]; Anal. Calcd for $\mathrm{C}_{18} \mathrm{H}_{21} \mathrm{~F}_{3} \mathrm{~N}_{2} \mathrm{O}_{2}$ : C, 61.01; H, 5.97; N, 7.91. Found: C, 60.80; H, 6.27; N, 7.55.

$\mathrm{N}$-Cyclohexyl-2-(4-hydroxyphenyl)-2-(2-oxoazetidin-1-yl)acetamide (1.6). White crystalline solid, Yield: 80\%; mp 176-177 ${ }^{\circ} \mathrm{C}$ (EtOAc/hexane); IR (KBr, $v_{\max }, \mathrm{cm}^{-1}$ ) 3321s, 2931m, 1716vs, $1655 \mathrm{vs}, 1544 \mathrm{~m}, 1515 \mathrm{~s}, 1227 \mathrm{~s} ;{ }^{1} \mathrm{H} \mathrm{NMR}\left(\mathrm{CDCl}_{3}, 300 \mathrm{MHz}, 30{ }^{\circ} \mathrm{C}\right): \delta_{\mathrm{H}} 7.26(\mathrm{~d}, J 8.8 \mathrm{~Hz}, 2 \mathrm{H})$, $6.78(\mathrm{~d}, J 8.8 \mathrm{~Hz}, 2 \mathrm{H}), 5.30(\mathrm{~s}, 1 \mathrm{H}), 4.80(\mathrm{~s}, 2 \mathrm{H}), 3.66(\mathrm{~m}, 1 \mathrm{H}), 3.52(\mathrm{td}, J 5.5,5.5,2.8 \mathrm{~Hz}, 1 \mathrm{H})$, 3.06 (td, $J 5.5,5.5,2.8 \mathrm{~Hz}, 1 \mathrm{H}), 2.92$ (ddd, $J 14.7,5.5,2.8 \mathrm{~Hz}, 1 \mathrm{H}), 2.79$ (ddd, $J 14.7,5.5,2.8 \mathrm{~Hz}$, 1H), $1.73(\mathrm{~m}, 5 \mathrm{H}), 1.19(\mathrm{~m}, 5 \mathrm{H}) ;{ }^{13} \mathrm{C} \mathrm{NMR}\left(\mathrm{CDCl}_{3}, 300 \mathrm{MHz}, 30{ }^{\circ} \mathrm{C}\right)$ : $\delta_{\mathrm{c}} 170.5$ (IV), 170.0 (IV), 130.6 (2C), 126.6 (IV), 116.6 (2C), 59.6, 50.0, 39.9, 36.1, 33.6, 33.5; HRMS m/z 302.1633 [M $\mathrm{C}_{17} \mathrm{H}_{22} \mathrm{~N}_{2} \mathrm{O}_{3}$ requires 302.1630]; Anal. Calcd for $\mathrm{C}_{17} \mathrm{H}_{22} \mathrm{~N}_{2} \mathrm{O}_{3}$ : C, 67.53; H, 7.33; N, 9.26. Found: C, 67.20; H, 7.23; N, 9.30.

$\mathrm{N}$-Cyclohexyl-2-(4-methoxyphenyl)-2-(2-oxoazetidin-1-yl)acetamide (1.7). White crystalline solid, Yield: 72\%; mp 114-115 ${ }^{\circ} \mathrm{C}$ (EtOAc/hexane); IR (KBr, $\left.v_{\max }, \mathrm{cm}^{-1}\right) 3318 \mathrm{~s}, 2930 \mathrm{~s}, 2853 \mathrm{~m}$, $1741 \mathrm{vs}, 1645 \mathrm{vs}, 1548 \mathrm{~s}, 1512 \mathrm{~s}, 1250 \mathrm{~s}, 1024 \mathrm{~m}, 802 \mathrm{~m} ;{ }^{1} \mathrm{H} \mathrm{NMR}\left(\mathrm{CDCl}_{3}, 400 \mathrm{MHz}, 30{ }^{\circ} \mathrm{C}\right): \delta_{\mathrm{H}}$ $7.28(\mathrm{~d}, J 8.8 \mathrm{~Hz}, 2 \mathrm{H}), 7.26(\mathrm{~d}, J 8.8 \mathrm{~Hz}, 2 \mathrm{H}), 6.27(\mathrm{br} \mathrm{d}, J 7.7 \mathrm{~Hz}, 1 \mathrm{H}), 5.33(\mathrm{~s}, 1 \mathrm{H}), 3.79(\mathrm{~s}, 3 \mathrm{H})$, $3.76(\mathrm{~m}, 1 \mathrm{H}), 3.62(\mathrm{td}, J 5.8,5.8,2.8 \mathrm{~Hz}, 1 \mathrm{H}), 3.15(\mathrm{td}, J 5.8,5.8,2.8 \mathrm{~Hz}, 1 \mathrm{H}), 2.98(\mathrm{ddd}, J 14.6$, $5.7,2.8 \mathrm{~Hz}, 1 \mathrm{H}), 2.83$ (ddd, $J 14.6,5.7,2.8 \mathrm{~Hz}, 1 \mathrm{H}), 1.88(\mathrm{~m}, 2 \mathrm{H}), 1.69(\mathrm{~m}, 3 \mathrm{H}), 1.38(\mathrm{~m}, 2 \mathrm{H})$, $1.18(\mathrm{~m}, 3 \mathrm{H}) ;{ }^{13} \mathrm{C} \mathrm{NMR}\left(\mathrm{CDCl}_{3}, 100 \mathrm{MHz}, 30{ }^{\circ} \mathrm{C}\right): \delta_{\mathrm{c}} 168.0$ (IV), 167.8 (IV), 159.7 (IV), 129.4 (2C), 127.1 (IV), 114.4 (2C), 59.2, 55.3, 48.6, 38.8, 36.1, 32.7, 25.5, 24.7, 24.7; HRMS m/z 316.1788 [M $\mathrm{M}^{+}, \mathrm{C}_{18} \mathrm{H}_{24} \mathrm{~N}_{2} \mathrm{O}_{3}$ requires 316.1787]; Anal. Calcd for $\mathrm{C}_{18} \mathrm{H}_{24} \mathrm{~N}_{2} \mathrm{O}_{3}$ : C, 68.33; H, 7.65; N, 8.85. Found: C, 67.95; H, 7.50; N, 8.98. 
$\mathrm{N}$-Cyclohexyl-2-(4-(dimethylamino)phenyl)-2-(2-oxoazetidin-1-yl)acetamide (1.8). Yellow crystalline solid; Yield 64\%; mp 122-125 ${ }^{\circ} \mathrm{C}$ (EtOAc/hexane); IR (KBr, $\left.v_{\max }, \mathrm{cm}^{-1}\right) 3275 \mathrm{~s}, 2930 \mathrm{~s}$, $1740 \mathrm{vs}, 1650 \mathrm{vs}, 1560 \mathrm{~m}, 1523 \mathrm{~s}, 1384 \mathrm{~m}, 1203 \mathrm{~m}, 800 \mathrm{~m} ;{ }^{1} \mathrm{H} \mathrm{NMR}\left(\mathrm{CDCl}_{3}, 400 \mathrm{MHz}, 30{ }^{\circ} \mathrm{C}\right): \delta_{\mathrm{H}}$ $7.25(\mathrm{~d}, J 8.8 \mathrm{~Hz}, 2 \mathrm{H}), 6.82(\mathrm{~d}, J 8.8 \mathrm{~Hz}, 2 \mathrm{H}), 6.00(\mathrm{~d}, J 8.0 \mathrm{~Hz}, 1 \mathrm{H}), 5.27(\mathrm{~s}, 1 \mathrm{H}), 3.81(\mathrm{~m}, 1 \mathrm{H})$, $3.610(\mathrm{td}, J 5.5,5.5,2.9 \mathrm{~Hz}, 1 \mathrm{H}), 3.15(\mathrm{td}, J 5.5,5.5,2.9 \mathrm{~Hz}, 1 \mathrm{H}), 2.99(\mathrm{~s}, 6 \mathrm{H}), 2.97(\mathrm{~m}, 1 \mathrm{H}), 2.87$ (ddd, $J 14.9,5.5,2.9 \mathrm{~Hz}, 1 \mathrm{H}), 1.92(\mathrm{~m}, 2 \mathrm{H}), 1.72(\mathrm{~m}, 3 \mathrm{H}), 1.37(\mathrm{~m}, 2 \mathrm{H}), 1.18(\mathrm{~m}, 3 \mathrm{H}) ;{ }^{13} \mathrm{C} \mathrm{NMR}$ $\left(\mathrm{CDCl}_{3}, 100 \mathrm{MHz}, 30{ }^{\circ} \mathrm{C}\right): \delta_{\mathrm{c}} 168.2$ (IV), 167.9 (IV), 149.7, 129.3, 123.9, 113.6, 59.4, 48.7, 41.1, 38.7, 36.1, 32.8, 25.5, 24.8, 24.7; HRMS m/z 343.2258 $\left[\mathrm{M}^{+}, \mathrm{C}_{20} \mathrm{H}_{29} \mathrm{~N}_{3} \mathrm{O}_{2}\right.$ requires 343.2260]; Anal. Calcd for $\mathrm{C}_{20} \mathrm{H}_{29} \mathrm{~N}_{3} \mathrm{O}_{2}$ : C, 69.94; H, 8.51; N, 12.23. Found: C, 69.84; H, 8.53; N, 12.51 .

N-Cyclohexyl-2-(2-oxoazetidin-1-yl)-2-(4-(prop-2-ynyloxy)phenyl)acetamide (1.9). White crystalline solid; Yield 81\%; IR (KBr, $\left.v_{\max }, \mathrm{cm}^{-1}\right) 3327 \mathrm{~s}, 2934 \mathrm{~m}, 1731 \mathrm{vs}, 1671 \mathrm{vs}, 1553 \mathrm{~m}, 1508 \mathrm{~s}$, $1222 \mathrm{~s}, 1024 \mathrm{~m} ;{ }^{1} \mathrm{H} \mathrm{NMR}\left(\mathrm{CDCl}_{3}, 400 \mathrm{MHz}, 30{ }^{\circ} \mathrm{C}\right): \delta_{\mathrm{H}} 7.30(\mathrm{~d}, J 8.5 \mathrm{~Hz}, 2 \mathrm{H}), 6.98(\mathrm{~d}, J 8.5 \mathrm{~Hz}$, 2H), 6.04 (br s, 1H), $5.29(\mathrm{~s}, 1 \mathrm{H}), 4.70(\mathrm{~d}, J 2.4 \mathrm{~Hz}, 2 \mathrm{H}), 3.78(\mathrm{~m}, 1 \mathrm{H}), 3.60(\mathrm{td}, J$ 5.6, 5.6, 2.7Hz, $1 \mathrm{H}), 3.15(\mathrm{td}, J 5.6,5.6,2.7 \mathrm{~Hz}, 1 \mathrm{H}), 2.97$ (ddd, $J$ 14.0, 5.6, $2.7 \mathrm{~Hz}, 1 \mathrm{H}), 2.86$ (ddd, $J$ 14.0, 5.6, $2.7 \mathrm{~Hz}, 1 \mathrm{H}), 2.54(\mathrm{t}, J 2.4 \mathrm{~Hz}, 1 \mathrm{H}), 1.88(\mathrm{~m}, 2 \mathrm{H}), 1.67(\mathrm{~m}, 3 \mathrm{H}), 1.33(\mathrm{~m}, 2 \mathrm{H}), 1.16(\mathrm{~m}, 3 \mathrm{H}) ;{ }^{13} \mathrm{C}$ NMR $\left(\mathrm{CDCl}_{3}, 100 \mathrm{MHz}, 30^{\circ} \mathrm{C}\right): \delta_{\mathrm{c}} 167.9$ (IV), 167.8 (IV), 157.7 (IV), 129.5, 128.0 (IV), 115.4, 78.3 (IV), 75.8 (IV), 59.5, 55.9, 48.7, 38.8, 36.2, 32.8, 25.4, 24.7, 24.7; HRMS m/z 340.1788 $\left[\mathrm{M}^{+}, \mathrm{C}_{20} \mathrm{H}_{24} \mathrm{~N}_{2} \mathrm{O}_{3}\right.$ requires 340.1787]; Anal. Calcd for $\mathrm{C}_{20} \mathrm{H}_{24} \mathrm{~N}_{2} \mathrm{O}_{3}: \mathrm{C}, 70.56 ; \mathrm{H}, 7.11 ; \mathrm{N}, 8.23$. Found: C, 70.42; H, 7.31; N, 8.27.

2-(2-Bromophenyl)- $\boldsymbol{N}$-cyclohexyl-2-(2-oxoazetidin-1-yl)acetamide (1.10). White crystalline solid, Yield: $79 \%$; mp 145-147 ${ }^{\circ} \mathrm{C}$ (EtOAc/hexane); IR (KBr, $v_{\max }, \mathrm{cm}^{-1}$ ) 3316s, 2934m, 1730vs, $1678 \mathrm{vs}, 1540 \mathrm{~s}, 1400 \mathrm{~m}, 1356 \mathrm{~m}, 1246 \mathrm{~m}, 1026 \mathrm{~m}, 727 \mathrm{~m} ;{ }^{1} \mathrm{H} \mathrm{NMR}\left(\mathrm{CDCl}_{3}, 300 \mathrm{MHz}, 30{ }^{\circ} \mathrm{C}\right): \delta_{\mathrm{H}}$ $7.62(\mathrm{dd}, J 8.0,1.1 \mathrm{~Hz}, 1 \mathrm{H}), 7.48(\mathrm{dd}, J 8.0,1.5 \mathrm{~Hz}, 1 \mathrm{H}), 7.33(\mathrm{td}, J 7.6,7.6,1.1 \mathrm{~Hz}, 1 \mathrm{H}), 7.22(\mathrm{td}$, $J$ 7.6, 7.6, 1.5Hz, 1H), 6.05 (br d, J 7.7Hz, 1H), $5.73(\mathrm{~s}, 1 \mathrm{H}), 3.79(\mathrm{~m}, 1 \mathrm{H}), 3.58$ (td, $J$ 5.5, 5.5, $2.6 \mathrm{~Hz}, 1 \mathrm{H}), 3.08(\mathrm{td}, J 5.5,5.5,2.6 \mathrm{~Hz}, 1 \mathrm{H}), 2.98(\mathrm{ddd}, J 14.6,5.5,2.6 \mathrm{~Hz}, 1 \mathrm{H}), 2.88$ (ddd, $J 14.6$, $5.5,2.6 \mathrm{~Hz}, 1 \mathrm{H}), 1.90(\mathrm{~m}, 2 \mathrm{H}), 1.63(\mathrm{~m}, 3 \mathrm{H}), 1.35(\mathrm{~m}, 2 \mathrm{H}), 1.26(\mathrm{~m}, 3 \mathrm{H}) ;{ }^{13} \mathrm{C} \mathrm{NMR}\left(\mathrm{CDCl}_{3}, 100\right.$ $\mathrm{MHz}, 30{ }^{\circ} \mathrm{C}$ ): $\delta_{\mathrm{c}} 167.6$ (IV), 167.8 (IV), 134.4 (IV), 133.4, 130.2, 129.8, 127.9, 124.67 (IV), 59.4, 48.8, 39.2, 36.3, 32.8, 32.7, 25.4, 24.7, 24.7; HRMS m/z 364.0789 [M $\mathrm{M}^{+}, \mathrm{C}_{17} \mathrm{H}_{21} \mathrm{BrN}_{2} \mathrm{O}_{2}$ requires 364.0786]; Anal. Calcd for $\mathrm{C}_{17} \mathrm{H}_{21} \mathrm{BrN}_{2} \mathrm{O}_{2}$ : C, 55.90; H, 5.79; N, 7.67. Found: C, 55.76; H, 5.60; N, 7.63.

$\mathrm{N}$-Cyclohexyl-2-(2-((dimethylamino)methyl)phenyl)-2-(2-oxoazetidin-1-yl)acetamide (1.11). Pale yellow crystalline solid; Yield $77 \% ;{ }^{1} \mathrm{H} \mathrm{NMR}\left(\mathrm{CDCl}_{3}, 300 \mathrm{MHz}, 30{ }^{\circ} \mathrm{C}\right): \delta_{\mathrm{H}} 7.77$ (d, $J$ $6.0 \mathrm{~Hz}, 1 \mathrm{H}), 7.50(\mathrm{dd}, J 8.2,2.1 \mathrm{~Hz}, 1 \mathrm{H}), 7.32(\mathrm{~m}, 1 \mathrm{H}), 7.17(\mathrm{~m}, 2 \mathrm{H}), 5.78(\mathrm{~s}, 1 \mathrm{H}), 4.20(\mathrm{~d}, J$ $14.0 \mathrm{~Hz}, 1 \mathrm{H}), 4.18(\mathrm{td}, J 5.5,5.5,2.6 \mathrm{~Hz}, 1 \mathrm{H}), 3.50(\mathrm{~m}, 2 \mathrm{H}), 2.98$ (ddd, $J 14.6,5.5,2.6 \mathrm{~Hz}, 1 \mathrm{H})$, $2.93(\mathrm{~d}, J 14.0 \mathrm{~Hz}, 1 \mathrm{H}), 2.77(\mathrm{ddd}, J 14.6,5.5,2.6 \mathrm{~Hz}, 1 \mathrm{H}), 2.24(\mathrm{~s}, 6 \mathrm{H}), 1.90(\mathrm{~m}, 2 \mathrm{H}), 1.58(\mathrm{~m}$, $3 \mathrm{H}), 1.25(\mathrm{~m}, 2 \mathrm{H}), 1.00(\mathrm{~m}, 3 \mathrm{H}) ;{ }^{13} \mathrm{C} \mathrm{NMR}\left(\mathrm{CDCl}_{3}, 75.5 \mathrm{MHz}, 30{ }^{\circ} \mathrm{C}\right): \delta_{\mathrm{c}} 169.0$ (IV), 167.5 (IV), 136.6 (IV), 131.3, 128.4, 127.7, 127.6, 62.9, 52.0, 47.8, 44.8 (2C), 40.5, 36.4, 33.2, 32.9, 25.5, 24.9, 24.7; HRMS m/z 343.2263 [ $\mathrm{M}^{+}, \mathrm{C}_{20} \mathrm{H}_{29} \mathrm{~N}_{3} \mathrm{O}_{2}$ requires 343.2260]; Anal. Calcd for $\mathrm{C}_{20} \mathrm{H}_{29} \mathrm{~N}_{3} \mathrm{O}_{2}$ : C, 69.94; H, 8.51; N, 12.23. Found: C, 70.01; H, 8.56; N, 12.03. 
2-(3-Bromophenyl)- $\boldsymbol{N}$-cyclohexyl-2-(2-oxoazetidin-1-yl)acetamide (1.12). White crystalline solid, Yield: 84\%; ${ }^{1} \mathrm{H}$ NMR $\left(\mathrm{CDCl}_{3}, 400 \mathrm{MHz}, 30^{\circ} \mathrm{C}\right): \delta_{\mathrm{H}} 7.50(\mathrm{~m}, 2 \mathrm{H}) ; 7.33(\mathrm{~m}, 1 \mathrm{H}), 7.25(\mathrm{~m}$, $1 \mathrm{H}), 6.31$ (br d, $J 7.6 \mathrm{~Hz}, 1 \mathrm{H}), 3.78(\mathrm{td}, J 5.6,5.6,2.8 \mathrm{~Hz}, 1 \mathrm{H}), 3.20(\mathrm{td}, J 5.6,5.6,2.8 \mathrm{~Hz}, 1 \mathrm{H})$, 3.01 (ddd, $J 14.6,5.6,2.8 \mathrm{~Hz}, 1 \mathrm{H}), 2.90$ (ddd, $J 14.6,5.6,2.8 \mathrm{~Hz}, 1 \mathrm{H}), 1.90(\mathrm{~m}, 2 \mathrm{H}), 1.65$ (m, $3 \mathrm{H}), 1.35(\mathrm{~m}, 2 \mathrm{H}), 1.15(\mathrm{~m}, 3 \mathrm{H}) ;{ }^{13} \mathrm{C} \mathrm{NMR}\left(\mathrm{CDCl}_{3}, 100 \mathrm{MHz}, 30{ }^{\circ} \mathrm{C}\right): \delta_{\mathrm{c}} 168.0$ (IV), 167.0 (IV), 137.2 (IV), 131.7, 131.2, 130.6, 126.7, 123.0 (IV), 59.5, 48.8, 39.2, 36.4, 32.7, 32.7, 25.4, 24.7, 24.7; HRMS $m / z$ 364.0786 $\left[\mathrm{M}^{+}, \mathrm{C}_{17} \mathrm{H}_{21} \mathrm{BrN}_{2} \mathrm{O}_{2}\right.$ requires 364.0786]; Anal. Calcd for $\mathrm{C}_{17} \mathrm{H}_{21} \mathrm{BrN}_{2} \mathrm{O}_{2}$ : C, 55.90; H, 5.79; N, 7.67. Found: C, 55.67; H, 5.83; N, 7.78.

$\mathrm{N}$-Cyclohexyl-2-(3-iodophenyl)-2-(2-oxoazetidin-1-yl)acetamide (1.13). White crystalline solid, Yield: $76 \%$; mp 106-107 ${ }^{\circ} \mathrm{C}\left(\right.$ EtOAc/hexane); IR (KBr, $\left.v_{\max }, \mathrm{cm}^{-1}\right) 3266 \mathrm{~s}, 2929 \mathrm{~s}, 2852 \mathrm{~m}$, 1744vs, 1651vs, 1564s, 1248m; ${ }^{1} \mathrm{H}$ NMR $\left(\mathrm{CDCl}_{3}, 300 \mathrm{MHz}, 30{ }^{\circ} \mathrm{C}\right): \delta_{\mathrm{H}} 7.67(\mathrm{~m}, 2 \mathrm{H}), 7.33(\mathrm{~d}, J$ $7.8 \mathrm{~Hz}, 1 \mathrm{H}), 7.10(\mathrm{t}, J 7.8 \mathrm{~Hz}, 1 \mathrm{H}), 6.33(\mathrm{br} \mathrm{d}, J 7.8 \mathrm{~Hz}, 1 \mathrm{H}), 5.29(\mathrm{~s}, 1 \mathrm{H}), 3.75(\mathrm{~m}, 1 \mathrm{H}), 3.63(\mathrm{td}, J$ $5.8,5.8,2.9 \mathrm{~Hz}, 1 \mathrm{H}), 3.20(\mathrm{td}, J 5.8,5.8,2.9 \mathrm{~Hz}, 1 \mathrm{H}), 3.00(\mathrm{ddd}, J 14.6,5.8,2.9 \mathrm{~Hz}, 1 \mathrm{H}), 2.88$ $(\mathrm{ddd}, J 14.6,5.8,2.9 \mathrm{~Hz}, 1 \mathrm{H}), 1.86(\mathrm{~m}, 2 \mathrm{H}), 1.62(\mathrm{~m}, 3 \mathrm{H}), 1.32(\mathrm{~m}, 2 \mathrm{H}), 1.13(\mathrm{~m}, 3 \mathrm{H}) ;{ }^{13} \mathrm{C} \mathrm{NMR}$ $\left(\mathrm{CDCl}_{3}, 75.5 \mathrm{MHz}, 30{ }^{\circ} \mathrm{C}\right): \delta_{\mathrm{c}} 167.9$ (IV), 166.9 (IV), 137.6, 137.2, 127.0, 130.6, 127.2, 94.7, 59.2, 48.7, 39.1, 36.3, 32.6, 25.4, 24.7, 24.6; HRMS $m / z 412.0465\left[\mathrm{M}^{+}, \mathrm{C}_{17} \mathrm{H}_{21} \mathrm{IN}_{2} \mathrm{O}_{2}\right.$ requires 412.0468]; Anal. Calcd for $\mathrm{C}_{17} \mathrm{H}_{21} \mathrm{IN}_{2} \mathrm{O}_{2}$ : C, 49.53; H, 5.13; N, 6.79. Found: C, 49.74; H, 5.17; $\mathrm{N}, 6.40$.

N-Cyclohexyl-2-(3-hydroxyphenyl)-2-(2-oxoazetidin-1-yl)acetamide (1.14). White crystalline solid, Yield: 79\%; mp 158-160 ${ }^{\circ} \mathrm{C}$ (EtOAc/hexane); IR (KBr, $\left.v_{\max }, \mathrm{cm}^{-1}\right) 3246 \mathrm{br}, 2934 \mathrm{~s}, 1726 \mathrm{vs}$, $1658 \mathrm{vs}, 1598 \mathrm{~s}, 1555 \mathrm{~m}, 1457 \mathrm{~s}, 1247 \mathrm{~m}, 1237 \mathrm{~m}, 735 \mathrm{~m} ;{ }^{1} \mathrm{H} \mathrm{NMR}\left(\mathrm{CDCl}_{3}, 300 \mathrm{MHz}, 30{ }^{\circ} \mathrm{C}\right): \delta_{\mathrm{H}}$ 8.19 (br s, 1H), $7.20(\mathrm{t}, J 7.9 \mathrm{~Hz}, 1 \mathrm{H}), 6.89(\mathrm{~m}, 1 \mathrm{H}), 6.83(\mathrm{~m}, 1 \mathrm{H}), 6.50(\mathrm{~d}, J 7.9 \mathrm{~Hz}, 1 \mathrm{H}), 5.31(\mathrm{~s}$, $1 \mathrm{H}), 3.71(\mathrm{~m}, 1 \mathrm{H}), 3.62(\mathrm{td}, J 5.5,5.5,2.7 \mathrm{~Hz}, 1 \mathrm{H}), 3.17(\mathrm{td}, J 5.5,5.5,2.7 \mathrm{~Hz}, 1 \mathrm{H}), 2.92$ (ddd, $J$ 14.7, 5.5, 2.7Hz, 1H), $2.79(\mathrm{ddd}, J 14.7,5.5,2.7 \mathrm{~Hz}, 1 \mathrm{H}), 1.83(\mathrm{~m}, 2 \mathrm{H}), 1.59(\mathrm{~m}, 3 \mathrm{H}), 1.26(\mathrm{~m}$, 2H), $1.11(\mathrm{~m}, 3 \mathrm{H}) ;{ }^{13} \mathrm{C} \mathrm{NMR}\left(\mathrm{CDCl}_{3}, 75.5 \mathrm{MHz}, 30^{\circ} \mathrm{C}\right): \delta_{\mathrm{c}} 168.1$ (IV), 157.4 (IV), 135.7, 130.3, $130.2,119.2,116.2,115.3,58.7,48.9,39.2,35.9,33.6,25.4,24.7,24.6,24.6$; HRMS m/z $302.1632\left[\mathrm{M}^{+}, \mathrm{C}_{17} \mathrm{H}_{22} \mathrm{~N}_{2} \mathrm{O}_{3}\right.$ requires 302.1630]; Anal. Calcd for $\mathrm{C}_{17} \mathrm{H}_{22} \mathrm{~N}_{2} \mathrm{O}_{3}$ : C, 67.53; H, 7.33; N, 9.26. Found: C, 67.42; H, 7.19; N, 9.36.

N-Cyclohexyl-2-(2-oxoazetidin-1-yl)-2-(3-(prop-2-ynyloxy)phenyl)acetamide (1.15). White crystalline solid; Yield 81\%; mp 128-129 ${ }^{\circ} \mathrm{C}$ (EtOAc/hexane); IR (KBr, $\left.v_{\max }, \mathrm{cm}^{-1}\right) 3264 \mathrm{~s}, 3216 \mathrm{~m}$, 2931s, 2852m, 1738vs, 1648vs, 1602m, 1269m, 1035m, 775m; ${ }^{1} \mathrm{H}$ NMR $\left(\mathrm{CDCl}_{3}, 300 \mathrm{MHz}, 30\right.$ $\left.{ }^{\circ} \mathrm{C}\right): \delta_{\mathrm{н}} 7.28(\mathrm{t}, J 7.5 \mathrm{~Hz}, 1 \mathrm{H}), 6.95(\mathrm{~m}, 3 \mathrm{H}), 6.18(\mathrm{~d}, J 7.7 \mathrm{~Hz}, 1 \mathrm{H}), 4.67(\mathrm{~d}, J 2.4 \mathrm{~Hz}, 2 \mathrm{H}), 3.75(\mathrm{~m}$, $1 \mathrm{H}), 3.62(\mathrm{td}, J 5.6,5.6,2.8 \mathrm{~Hz}, 1 \mathrm{H}), 3.19$ (td, $J 5.6,5.6,2.8 \mathrm{~Hz}, 1 \mathrm{H}), 2.97$ (ddd, $J 14.7,5.6$, $2.8 \mathrm{~Hz}, 1 \mathrm{H}), 2.84(\mathrm{ddd}, J 14.7,5.6,2.8 \mathrm{~Hz}, 1 \mathrm{H}), 2.51(\mathrm{t}, J 2.4 \mathrm{~Hz}, 1 \mathrm{H}), 1.85(\mathrm{~m}, 2 \mathrm{H}), 1.62(\mathrm{~m}, 3 \mathrm{H})$, $1.30(\mathrm{~m}, 2 \mathrm{H}), 1.11(\mathrm{~m}, 3 \mathrm{H}) ;{ }^{13} \mathrm{C} \mathrm{NMR}\left(\mathrm{CDCl}_{3}, 75.5 \mathrm{MHz}, 30{ }^{\circ} \mathrm{C}\right): \delta_{\mathrm{c}} 167.8(\mathrm{IV}), 167.3(\mathrm{IV})$, 157.9 (IV), 135.5, 130.1, 121.2, 114.9, 114.7, 78.3 (IV), 75.7, 59.8, 55.8, 48.6, 39.0, 36.21, 32.7, 25.4, 24.7, 24.6; HRMS m/z 302.163340.1783 [M $\mathrm{M}^{+}, \mathrm{C}_{20} \mathrm{H}_{24} \mathrm{~N}_{2} \mathrm{O}_{3}$ requires 340.1787]; Anal. Calcd for $\mathrm{C}_{20} \mathrm{H}_{24} \mathrm{~N}_{2} \mathrm{O}_{3}$ : C, 70.23; H, 7.00; N, 7.90. Found: C, 70.42; H, 7.31; N, 8.27.

$\mathbf{N}$-Cyclohexyl-2-(2,4-dichlorophenyl)-2-(2-oxoazetidin-1-yl)acetamide (1.16). White crystalline solid; Yield 77\%; mp 156-157 ${ }^{\circ} \mathrm{C}$ (EtOAc/hexane); IR (KBr, $\left.v_{\max }, \mathrm{cm}^{-1}\right) 3292 \mathrm{~m}, 2933 \mathrm{~s}$, 
$1741 \mathrm{vs}, 1647 \mathrm{vs}, 1553 \mathrm{~s}, 1473 \mathrm{~m}, 1252 \mathrm{~m}, 812 \mathrm{~m}, 569 \mathrm{~m} ;{ }^{1} \mathrm{H} \mathrm{NMR}\left(\mathrm{CDCl}_{3}, 400 \mathrm{MHz}, 30{ }^{\circ} \mathrm{C}\right): \delta_{\mathrm{H}}$ $7.46(\mathrm{~m}, 2 \mathrm{H}), 7.29(\mathrm{~m}, 1 \mathrm{H}), 6.30$ (br d, J 7.7Hz, 1H), $5.72(\mathrm{~s}, 1 \mathrm{H}), 3.82(\mathrm{~m}, 1 \mathrm{H}), 3.60$ (td, J 5.5, $5.5,2.8 \mathrm{~Hz}, 1 \mathrm{H}), 3.12(\mathrm{td}, J 5.5,5.5,2.8 \mathrm{~Hz}, 1 \mathrm{H}), 3.02(\mathrm{ddd}, J 14.6,5.5,2.6 \mathrm{~Hz}), 2.92(\mathrm{ddd}, J 14.6$, $5.5,2.6 \mathrm{~Hz}), 1.92(\mathrm{~m}, 2 \mathrm{H}), 1.73(\mathrm{~m}, 3 \mathrm{H}), 1.41(\mathrm{~m}, 2 \mathrm{H}), 1.21(\mathrm{~m}, 3 \mathrm{H}) ;{ }^{13} \mathrm{C} \mathrm{NMR}\left(\mathrm{CDCl}_{3}, 100\right.$ $\mathrm{MHz}, 30{ }^{\circ} \mathrm{C}$ ): $\delta_{\mathrm{c}} 167.7$ (IV), 166.7 (IV), 135.4, 135.9, 131.3, 129.9, 127.6, 70.7, 56.4, 48.8, 39.2, 36.2, 32.7, 32.7, 25.4, 24.7, 24. 7; HRMS $m / z$ 354.0904 $\left[\mathrm{M}^{+}, \mathrm{C}_{17} \mathrm{H}_{20} \mathrm{Cl}_{2} \mathrm{~N}_{2} \mathrm{O}_{2}\right.$ requires 354.0902]; Anal. Calcd for $\mathrm{C}_{17} \mathrm{H}_{20} \mathrm{Cl}_{2} \mathrm{~N}_{2} \mathrm{O}_{2}$ : C, 57.47; H, 5.67; N, 7.89. Found: C, 57.10; H, 5.71; N, 7.91.

$\mathbf{N}$-Cyclohexyl-2-(2,4-difluorophenyl)-2-(2-oxoazetidin-1-yl)acetamide $\quad$ (1.17). White crystalline solid; Yield 84\%; mp 147-150 ${ }^{\circ} \mathrm{C}$ (EtOAc/hexane); IR (KBr, $\left.v_{\max }, \mathrm{cm}^{-1}\right) 3315 \mathrm{~s}, 2932 \mathrm{~s}$, $2855 \mathrm{~m}, 1748 \mathrm{vs}, 1673 \mathrm{vs}, 1531 \mathrm{~s}, 1505 \mathrm{~s}, 1392 \mathrm{~m}, 1248 \mathrm{~m}, 1141 \mathrm{~m} ;{ }^{1} \mathrm{H}$ NMR $\left(\mathrm{CDCl}_{3}, 400 \mathrm{MHz}, 30\right.$ $\left.{ }^{\circ} \mathrm{C}\right): \delta_{\text {н }} 7.45(\mathrm{dd}, J 14.7,8.4 \mathrm{~Hz}, 1 \mathrm{H}), 6.88(\mathrm{dt}, J 11.2,5.7 \mathrm{~Hz}, 2 \mathrm{H}), 6.27(\mathrm{br} \mathrm{d}, J 7.2 \mathrm{~Hz}, 1 \mathrm{H}), 5.56$ $(\mathrm{s}, 1 \mathrm{H}), 3.77(\mathrm{~m}, 1 \mathrm{H}), 3.59(\mathrm{~m}, 1 \mathrm{H}), 3.17(\mathrm{~m}, 1 \mathrm{H}), 3.00(\mathrm{ddd}, J 14.8,5.6,2.8 \mathrm{~Hz}, 1 \mathrm{H}), 2.90$ (ddd, $J$ 14.8, 5.6, 2.8Hz, 1H), $1.85(\mathrm{~m}, 2 \mathrm{H}), 1.64(\mathrm{~m}, 3 \mathrm{H}), 1.35(\mathrm{~m}, 2 \mathrm{H}), 1.15(\mathrm{~m}, 3 \mathrm{H}) ;{ }^{13} \mathrm{C}$ NMR $\left(\mathrm{CDCl}_{3}, 400 \mathrm{MHz}, 30{ }^{\circ} \mathrm{C}\right): \delta_{\mathrm{c}} 167.8$ (IV), 166.6 (IV), 131.2, 130.1, 112.0, 111.8, 104.7, 104.4, 53.6, 48.8, 39.2, 36.4, 32.7, 25.4, 24.7, 24.7; HRMS $m / z$ 322.1491 $\left[\mathrm{M}^{+}, \mathrm{C}_{17} \mathrm{H}_{20} \mathrm{~F}_{2} \mathrm{~N}_{2} \mathrm{O}_{2}\right.$ requires 322.1493]; Anal. Calcd for $\mathrm{C}_{17} \mathrm{H}_{20} \mathrm{~F}_{2} \mathrm{~N}_{2} \mathrm{O}_{2}$ : C, 63.34; H, 6.25; N, 8.69. Found: C, 63.00; H, 6.33; N, 8.71.

N-Cyclohexyl-2-(3,4-dichlorophenyl)-2-(2-oxoazetidin-1-yl)acetamide (1.18). White crystalline solid; Yield 84\%; mp 117-118 ${ }^{\circ} \mathrm{C}$ (EtOAc/hexane); IR (KBr, $\left.v_{\max }, \mathrm{cm}^{-1}\right) 3303 \mathrm{~s}, 2932 \mathrm{~s}$, 2854m, 1741vs, 1656vs, 1542m, 1032m; ${ }^{1} \mathrm{H} \mathrm{NMR}\left(\mathrm{CDCl}_{3}, 400 \mathrm{MHz}, 30{ }^{\circ} \mathrm{C}\right): \delta_{\mathrm{H}} 7.43(\mathrm{~d}, \mathrm{~J} 2.0 \mathrm{~Hz}$, $1 \mathrm{H}), 7.41(\mathrm{~d}, J 7.8 \mathrm{~Hz}, 1 \mathrm{H}), 7.21(\mathrm{~d}, J 7.8 \mathrm{~Hz}, 1 \mathrm{H}), 6.85(\mathrm{br} \mathrm{s}, 1 \mathrm{H}), 5.41(\mathrm{~s}, 1 \mathrm{H}), 3.70(\mathrm{~m}, 2 \mathrm{H})$, $3.24(\mathrm{td}, J 5.5,5.5,2.6 \mathrm{~Hz}, 1 \mathrm{H}), 3.00(\mathrm{ddd}, J 14.5,5.5,2.6 \mathrm{~Hz}, 1 \mathrm{H}), 2.90$ (ddd, $J 14.5,5.5,2.6 \mathrm{~Hz}$, $1 \mathrm{H}), 2.29(\mathrm{~m}, 1 \mathrm{H}), 1.89(\mathrm{~m}, 2 \mathrm{H}), 1.69(\mathrm{~m}, 3 \mathrm{H}), 1.39(\mathrm{~m}, 2 \mathrm{H}), 1.16(\mathrm{~m}, 3 \mathrm{H}),{ }^{13} \mathrm{C} \mathrm{NMR}\left(\mathrm{CDCl}_{3}\right.$, $100 \mathrm{MHz}, 30{ }^{\circ} \mathrm{C}$ ): $\delta_{\mathrm{c}} 168.0$ (IV), 166.8 (IV), 135.4, 133.1, 132.8, 130.9, 130.0, 127.3, 58.3, 58.2, 48.7, 39.3, 32.6, 25.4, 24.7, 23.6; HRMS $m / z$ 354.0900 $\left[\mathrm{M}^{+}, \mathrm{C}_{17} \mathrm{H}_{20} \mathrm{Cl}_{2} \mathrm{~N}_{2} \mathrm{O}_{2}\right.$ requires 354.0902]; Anal. Calcd for $\mathrm{C}_{17} \mathrm{H}_{20} \mathrm{Cl}_{2} \mathrm{~N}_{2} \mathrm{O}_{2}$ : C, 57.47; H, 5.67; N, 7.89. Found: C, 57.77; H, 5.90; N, 7.53.

2-(2-Chloro-3,6-difluorophenyl)- $\mathbf{N}$-cyclohexyl-2-(2-oxoazetidin-1-yl)acetamide (1.19). White crystalline solid; Yield 79\%; mp 182-184 ${ }^{\circ} \mathrm{C}$ (EtOAc/hexane); IR (KBr, $\left.v_{\max }, \mathrm{cm}^{-1}\right) 3339 \mathrm{~m}$, $2933 \mathrm{~m}, 1751 \mathrm{vs}, 1667 \mathrm{vs}, 1529 \mathrm{~s}, 1470 \mathrm{~s}, 1388 \mathrm{~m}, 1257 \mathrm{~m}, 1228 \mathrm{~m}, 821 \mathrm{~m} ;{ }^{1} \mathrm{H} \mathrm{NMR}\left(\mathrm{CDCl}_{3}, 300\right.$ $\left.\mathrm{MHz}, 30{ }^{\circ} \mathrm{C}\right): \delta_{\mathrm{H}} 7.21(\mathrm{~m}, 1 \mathrm{H}), 7.07(\mathrm{~m}, 1 \mathrm{H}), 6.00(\mathrm{~s}, 1 \mathrm{H}), 5.98(\mathrm{~d}, J 6.8 \mathrm{~Hz}, 1 \mathrm{H}), 3.88(\mathrm{~m}, 1 \mathrm{H})$, $3.59(\mathrm{td}, J 5.5,5.5,2.6 \mathrm{~Hz}, 1 \mathrm{H}), 3.14(\mathrm{~m}, 2 \mathrm{H}), 2.94$ (ddd, $J 14.5,5.5,2.6 \mathrm{~Hz}, 1 \mathrm{H}), 1.96(\mathrm{~m}, 2 \mathrm{H})$, $1.72(\mathrm{~m}, 3 \mathrm{H}), 1.40(\mathrm{~m}, 2 \mathrm{H}), 1.23(\mathrm{~m}, 3 \mathrm{H}) ;{ }^{13} \mathrm{C} \mathrm{NMR}\left(\mathrm{CDCl}_{3}, 75.5 \mathrm{MHz}, 30{ }^{\circ} \mathrm{C}\right): \delta_{\mathrm{c}} 168.2$ (IV), 165.5 (IV), 117.3, 117.0, 115.2, 115.1, 114.9, 114.8, 53.1, 48.9, 39.4, 36.8, 32.9, 32.7, 25.5, 24.7; HRMS $m / z \quad 356.1100 \quad\left[\mathrm{M}^{+}, \quad \mathrm{C}_{17} \mathrm{H}_{19} \mathrm{ClF}_{2} \mathrm{~N}_{2} \mathrm{O}_{2}\right.$ requires 356.1103]; Anal. Calcd for $\mathrm{C}_{17} \mathrm{H}_{19} \mathrm{ClF}_{2} \mathrm{~N}_{2} \mathrm{O}_{2}$ : C, 57.23; H, 5.37; N, 7.85. Found: C, 57.13; H, 5.59; N, 7.87.

\section{$\mathrm{N}$-Cyclohexyl-2-(2-fluoro-4-(trifluoromethyl)phenyl)-2-(2-oxoazetidin-1-yl)acetamide}

(1.20). White crystalline solid; Yield $74 \%$; mp 108-109 ${ }^{\circ} \mathrm{C}$ (EtOAc/hexane); ${ }^{1} \mathrm{H} \mathrm{NMR}\left(\mathrm{CDCl}_{3}\right.$, $\left.300 \mathrm{MHz}, 30{ }^{\circ} \mathrm{C}\right): \delta_{\mathrm{H}} 7.57(\mathrm{t}, J 7.6 \mathrm{~Hz}, 1 \mathrm{H}), 7.44(\mathrm{~d}, J 8.2 \mathrm{~Hz}, 1 \mathrm{H}), 7.37(\mathrm{~d}, J 9.9 \mathrm{~Hz}, 1 \mathrm{H}), 6.12(\mathrm{~d}$, $J 7.9 \mathrm{~Hz}, 1 \mathrm{H}), 5.55(\mathrm{~s}, 1 \mathrm{H}), 3.82(\mathrm{~m}, 1 \mathrm{H}), 3.58(\mathrm{td}, J 5.7,5.7,3.0 \mathrm{~Hz}, 1 \mathrm{H}), 3.22(\mathrm{td}, J 5.7,5.7$, $3.0 \mathrm{~Hz}, 1 \mathrm{H}), 3.03(\mathrm{ddd}, J 14.8,5.7,3.0 \mathrm{~Hz}, 1 \mathrm{H}), 2.94(\mathrm{ddd}, J 14.8,5.7,3.0 \mathrm{~Hz}, 1 \mathrm{H}), 2.01(\mathrm{~m}, 2 \mathrm{H})$, 
$1.70(\mathrm{~m}, 3 \mathrm{H}), 1.36(\mathrm{~m}, 2 \mathrm{H}), 1.21(\mathrm{~m}, 3 \mathrm{H})$; HRMS $m / z 372.1460\left[\mathrm{M}^{+}, \mathrm{C}_{18} \mathrm{H}_{20} \mathrm{~F}_{4} \mathrm{~N}_{2} \mathrm{O}_{2}\right.$ requires 372.1461]; Anal. Calcd for $\mathrm{C}_{18} \mathrm{H}_{20} \mathrm{~F}_{4} \mathrm{~N}_{2} \mathrm{O}_{2}$ : C, 58.06; H, 5.41; N, 7.52. Found: C, 57.93; H, 5.47; $\mathrm{N}, 7.72$.

N-Cyclohexyl-2-(3,4-dimethoxyphenyl)-2-(2-oxoazetidin-1-yl)acetamide (1.21). White crystalline solid; Yield 73\%; mp 140-141 ${ }^{\circ} \mathrm{C}$ (EtOAc/hexane); IR (KBr, $\left.v_{\max }, \mathrm{cm}^{-1}\right) 3275 \mathrm{~s}, 2924 \mathrm{~s}$, $2853 \mathrm{~m}, 1752 \mathrm{vs}, 1644 \mathrm{~s}, 1517 \mathrm{~s}, 1260 \mathrm{~s}, 1250 \mathrm{~s}, 1145 \mathrm{~m}, 1027 \mathrm{~m} ;{ }^{1} \mathrm{H}$ NMR $\left(\mathrm{CDCl}_{3}, 300 \mathrm{MHz}, 30\right.$ $\left.{ }^{\circ} \mathrm{C}\right): \delta_{\text {н }} 6.89(\mathrm{~m}, 3 \mathrm{H}), 5.89(\mathrm{~d}, J 7.8 \mathrm{~Hz}, 1 \mathrm{H}), 5.22(\mathrm{~s}, 1 \mathrm{H}), 3.88(\mathrm{~s}, 3 \mathrm{H}), 3.86(\mathrm{~s}, 3 \mathrm{H}), 3.80(\mathrm{~m}, 1 \mathrm{H})$, $3.62(\mathrm{td}, J 5.8,5.8,2.7 \mathrm{~Hz}, 1 \mathrm{H}), 3.16(\mathrm{td}, J 5.8,5.8,2.7 \mathrm{~Hz}, 1 \mathrm{H}), 3.04(\mathrm{ddd}, J 14.6,5.8,2.7 \mathrm{~Hz}$, 1H), 2.89 (ddd, J 14.6, 5.8, 2.7Hz, 1H), $1.93(\mathrm{~m}, 2 \mathrm{H}), 1.71(\mathrm{~m}, 3 \mathrm{H}), 1.43(\mathrm{~m}, 2 \mathrm{H}), 1.19(\mathrm{~m}, 3 \mathrm{H})$; ${ }^{13} \mathrm{C} \mathrm{NMR}\left(\mathrm{CDCl}_{3}, 100 \mathrm{MHz}, 30{ }^{\circ} \mathrm{C}\right): \delta_{\mathrm{c}} 167.3$ (IV), 162.8 (IV), 127.3 (IV), 120.7, 111.5, 111.3, 60.0, 56.0, 56.0, 48.7, 38.8, 36.2, 32.8, 25.5, 24.7; HRMS m/z 346.1895 [M ${ }^{+}, \mathrm{C}_{19} \mathrm{H}_{26} \mathrm{~N}_{2} \mathrm{O}_{4}$ requires 346.1893]; Anal. Calcd for $\mathrm{C}_{19} \mathrm{H}_{26} \mathrm{~N}_{2} \mathrm{O}_{4}$ : C, 65.87; H, 7.56; N, 8.09. Found: C, 65.55; H, $7.35 ; \mathrm{N}, 8.02$.

N-Cyclohexyl-2-(4-hydroxy-3-methoxyphenyl)-2-(2-oxoazetidin-1-yl)acetamide

(1.22). White crystalline solid, Yield: $76 \%$; mp $178-180{ }^{\circ} \mathrm{C}\left(\right.$ EtOAc/hex); $\left(\mathrm{KBr}, v_{\max }, \mathrm{cm}^{-1}\right) 3489 \mathrm{~s}$, $3283 \mathrm{~m}, 2938 \mathrm{~m}, 1741 \mathrm{vs}, 1647 \mathrm{vs}, 1519 \mathrm{~m}, 1270 \mathrm{~m}, 1263 \mathrm{~m}, 1251 \mathrm{~m}, 1032 \mathrm{~m} ;{ }^{1} \mathrm{H} \mathrm{NMR}\left(\mathrm{CDCl}_{3}, 300\right.$ $\left.\mathrm{MHz}, 30{ }^{\circ} \mathrm{C}\right): \delta_{\text {н }} 6.92(\mathrm{~m}, 3 \mathrm{H}), 5.86(\mathrm{~d}, J 7.8 \mathrm{~Hz}, 1 \mathrm{H}), 5.70(\mathrm{~s}, 1 \mathrm{H}), 5.20(\mathrm{~s}, 1 \mathrm{H}), 3.88(\mathrm{~s}, 3 \mathrm{H})$, $3.82(\mathrm{~m}, 1 \mathrm{H}), 3.61(\mathrm{td}, J 5.8,5.8,2.8 \mathrm{~Hz}, 1 \mathrm{H}), 3.16(\mathrm{td}, J 5.8,5.8,2.8 \mathrm{~Hz}, 1 \mathrm{H}), 3.03(\mathrm{ddd}, J 14.6$, $5.8,2.9 \mathrm{~Hz}, 1 \mathrm{H}), 2.89$ (ddd, $J 14.6,5.8,2.9 \mathrm{~Hz}, 1 \mathrm{H}), 1.91(\mathrm{~m}, 2 \mathrm{H}), 1.71(\mathrm{~m}, 3 \mathrm{H}), 1.42(\mathrm{~m}, 2 \mathrm{H})$, $1.21(\mathrm{~m}, 3 \mathrm{H}) ;{ }^{13} \mathrm{C} \mathrm{NMR}\left(\mathrm{CDCl}_{3}, 75.5 \mathrm{MHz}, 30{ }^{\circ} \mathrm{C}\right): \delta_{\mathrm{c}} 167.9$ (IV), 167.8 (IV), 146.9 (IV), 146.0 (IV), 126.6 (IV), 121.3, 114.8, 110.8, 59.7, 56.0, 48. 6, 38.8, 36.0, 32.6 (2C), 25.4, 24.7, 24.6; IR HRMS $m / z 332.1739\left[\mathrm{M}^{+}, \mathrm{C}_{18} \mathrm{H}_{24} \mathrm{~N}_{2} \mathrm{O}_{4}\right.$ requires 332.1736]; Anal. Calcd for $\mathrm{C}_{18} \mathrm{H}_{24} \mathrm{~N}_{2} \mathrm{O}_{4}$ : C, 65.04; H, 7.28; N, 8.43. Found: C, 64.87; H, 7.22; N, 8.25.

$\mathrm{N}$-Cyclohexyl-2-(2,3-dimethoxyphenyl)-2-(2-oxoazetidin-1-yl)acetamide (1.23). White crystalline solid, Yield: $72 \%$; mp 148-149 ${ }^{\circ} \mathrm{C}$ (EtOAc/hex); IR (KBr, $\left.v_{\max }, \mathrm{cm}^{-1}\right) 3255 \mathrm{~s}, 2930 \mathrm{~s}$, 2852m, 1747vs, 1650vs, 1557s, 1483s, 1274s, 1068s, 742m; ${ }^{1} \mathrm{H} \mathrm{NMR}\left(\mathrm{CDCl}_{3}, 400 \mathrm{MHz}, 30{ }^{\circ} \mathrm{C}\right)$ : $\delta_{\mathrm{H}} 7.06(\mathrm{t}, J 8.0 \mathrm{~Hz}, 1 \mathrm{H}), 6.96(\mathrm{~d}, J 8.0 \mathrm{~Hz}, 1 \mathrm{H}), 6.92(\mathrm{~d}, J 8.0 \mathrm{~Hz}, 1 \mathrm{H}), 5.85(\mathrm{br} \mathrm{s}, 1 \mathrm{H}), 3.88(\mathrm{~s}$, $3 \mathrm{H}), 3.87(\mathrm{~s}, 3 \mathrm{H}), 3.73(\mathrm{~m}, 1 \mathrm{H}), 3.67(\mathrm{td}, J 5.6,5.6,2.8 \mathrm{~Hz}, 1 \mathrm{H}), 3.23(\mathrm{td}, J 5.6,5.6,2.8 \mathrm{~Hz}, 1 \mathrm{H})$, $2.98(\mathrm{ddd}, J 14.0,5.6,2.8 \mathrm{~Hz}, 1 \mathrm{H}), 2.85(\mathrm{ddd}, J 14.0,5.6,2.8 \mathrm{~Hz}, 1 \mathrm{H}), 1.85(\mathrm{~m}, 2 \mathrm{H}), 1.63(\mathrm{~m}$, $3 \mathrm{H}), 1.33(\mathrm{~m}, 2 \mathrm{H}), 1.08(\mathrm{~m}, 3 \mathrm{H}) ;{ }^{13} \mathrm{C} \mathrm{NMR}\left(\mathrm{CDCl}_{3}, 75.5 \mathrm{MHz}, 30{ }^{\circ} \mathrm{C}\right): \delta_{\mathrm{c}} 168.0(\mathrm{IV}, \mathrm{CO}), 167.9$ (IV, CO), 152.8, 146.8, 129.2, 120.7, 112.8, 61.0, 55.8, 54.1, 48.6, 39.5, 32.9, 32.7, 25.5, 24.8, 24.9; HRMS $m / z$ 346.1891 [ $\mathrm{M}^{+}, \mathrm{C}_{19} \mathrm{H}_{26} \mathrm{~N}_{2} \mathrm{O}_{4}$ requires 346.1893]; Anal. Calcd for $\mathrm{C}_{19} \mathrm{H}_{26} \mathrm{~N}_{2} \mathrm{O}_{4}$ : C, 65.87; H, 7.56; N, 8.09. Found: C, 65.65; H, 7.54; N, 8.07.

$\mathrm{N}$-Cyclohexyl-2-(2,4-dimethoxyphenyl)-2-(2-oxoazetidin-1-yl)acetamide (1.24). White crystalline solid; Yield 82\%; mp 103-105 ${ }^{\circ} \mathrm{C}$ (EtOAc/hexane); IR (KBr, $\left.v_{\max }, \mathrm{cm}^{-1}\right) 3287 \mathrm{~s}, 2934 \mathrm{~s}$, $1748 \mathrm{vs}, 1651 \mathrm{vs}, 1615 \mathrm{~s}, 1506 \mathrm{~s}, 1209 \mathrm{~s}, 1039 \mathrm{~m} ;{ }^{1} \mathrm{H} \mathrm{NMR}\left(\mathrm{CDCl}_{3}, 400 \mathrm{MHz}, 30{ }^{\circ} \mathrm{C}\right): \delta_{\mathrm{H}} 7.25(\mathrm{~d}, J$ $8.0 \mathrm{~Hz}, 1 \mathrm{H}), 6.49(\mathrm{~m}, 2 \mathrm{H}), 5.71(\mathrm{~d}, \mathrm{~J} 8.0 \mathrm{~Hz}, 1 \mathrm{H}), 5.55(\mathrm{~s}, 1 \mathrm{H}), 3.81(\mathrm{~s}, 3 \mathrm{H}), 3.81(\mathrm{~s}, 3 \mathrm{H}), 3.78$ $(\mathrm{m}, 1 \mathrm{H}), 3.61(\mathrm{td}, J 5.6,5.6,2.8 \mathrm{~Hz}, 1 \mathrm{H}), 3.13(\mathrm{td}, J 5.6,5.6,2.8 \mathrm{~Hz}, 1 \mathrm{H}), 2.98(\mathrm{ddd}, J 14.6,5.7$, $2.7 \mathrm{~Hz}, 1 \mathrm{H}), 2.85$ (ddd, J 14.6, 5.7, $2.7 \mathrm{~Hz}, 1 \mathrm{H}), 1.91$ (m, 2H), 1.72 (m, 3H), 1.4 (m, 2H), 1.19 $(\mathrm{m}, 3 \mathrm{H}) ;{ }^{13} \mathrm{C} \mathrm{NMR}\left(\mathrm{CDCl}_{3}, 100 \mathrm{MHz}, 30{ }^{\circ} \mathrm{C}\right): \delta_{\mathrm{c}} 168.2$ (IV), 168.0 (IV), 161.3 (IV), 158.1 (IV), 
130.5, 115.9 (IV), 104.7, 98.9, 55.4, 55.4, 54.3, 48.4, 39.2, 36.1, 32.9, 32.8, 25.5, 24.7, 24.7; HRMS m/z $346.1894\left[\mathrm{M}^{+}, \mathrm{C}_{19} \mathrm{H}_{26} \mathrm{~N}_{2} \mathrm{O}_{4}\right.$ requires 346.1893]; Anal. Calcd for $\mathrm{C}_{19} \mathrm{H}_{26} \mathrm{~N}_{2} \mathrm{O}_{4}$ : C, 65.87; H, 7.56; N, 8.09. Found: C, 65.5; H, 7.56; N, 8.26.

N-Cyclohexyl-2-(2-oxoazetidin-1-yl)-2-(2,3,4-trimethoxyphenyl)acetamide (1.25). White crystalline solid, Yield: $75 \%$; mp $125-127^{\circ} \mathrm{C}$ (EtOAc/hex); IR (KBr, $\left.v_{\max }, \mathrm{cm}^{-1}\right) 3255 \mathrm{~m}, 2926 \mathrm{~s}$, 2852m, 1745vs, 1643vs, 1494s, 1468s, 1096vs, 1040s; ${ }^{1} \mathrm{H}$ NMR $\left(\mathrm{CDCl}_{3}, 400 \mathrm{MHz}, 30{ }^{\circ} \mathrm{C}\right): \delta_{\mathrm{H}}$ 7.06 (d, J $9 \mathrm{~Hz}, 1 \mathrm{H}), 6.67$ (d, J $9 \mathrm{~Hz}, 1 \mathrm{H}), 5.85$ (d, J $9 \mathrm{~Hz}, 1 \mathrm{H}), 5.56(\mathrm{~s}, 1 \mathrm{H}), 3.92$ (s, 3H), 3.87 $(\mathrm{s}, 3 \mathrm{H}), 3.87(\mathrm{~s}, 3 \mathrm{H}), 3.80(\mathrm{~m}, 1 \mathrm{H}), 3.64(\mathrm{td}, J 5.7,5.7,2.7 \mathrm{~Hz}, 1 \mathrm{H}), 3.21(\mathrm{td}, J$ 5.7, 5.7, $2.7 \mathrm{~Hz}$, 1H), 3.01 (ddd, $J$ 14.6, 5.6, $2.6 \mathrm{~Hz}, 1 \mathrm{H}), 2.87$ (ddd, $J$ 14.6, 5.6, 2.6 Hz, 1H), 1.93 (m, 2H), 1.73 (m, 3H), 1.39 (m, 2H), $1.22(\mathrm{~m}, 3 \mathrm{H}) ;{ }^{13} \mathrm{C} \mathrm{NMR}\left(\mathrm{CDCl}_{3}, 75.5 \mathrm{MHz}, 30{ }^{\circ} \mathrm{C}\right): \delta_{\mathrm{c}} 168.0(\mathrm{IV}), 167.8$ (IV), 154.1 (IV), 151.5 (IV), 142.0 (IV), 123.6, 121.0 (IV), 107.2, 61.0, 60.6, 55.8, 54.3, 48.4, 39.2, 36.0, 32.8, 32.6, 25.3, 24.6, 24.6; HRMS m/z 376.1999 [ $\left[\mathrm{M}^{+}, \mathrm{C}_{20} \mathrm{H}_{28} \mathrm{~N}_{2} \mathrm{O}_{5}\right.$ requires 376.1998]; Anal. Calcd for $\mathrm{C}_{20} \mathrm{H}_{28} \mathrm{~N}_{2} \mathrm{O}_{5}$ : C, 63.21; H, 7.5; N, 7.44. Found: C, 63.81; H, 7.3; N, 7.46 .

$\mathrm{N}$-Cyclohexyl-2-(2-oxoazetidin-1-yl)-2-(ferrocenyl)acetamide (1.26). Orange crystalline solid; Yield 67\%; mp 145-146 ${ }^{\circ} \mathrm{C}$ (EtOAc/hexane); IR (KBr, $\left.v_{\max }, \mathrm{cm}^{-1}\right) 3332 \mathrm{~s}, 2930 \mathrm{~s}, 2853 \mathrm{~m}, 1736 \mathrm{vs,}$ $1671 \mathrm{vs}, 1544 \mathrm{~s}, 1396 \mathrm{~m}, 1229 \mathrm{~m}, 486 \mathrm{~s} ;{ }^{1} \mathrm{H} \mathrm{NMR}\left(\mathrm{CDCl}_{3}, 400 \mathrm{MHz}, 30{ }^{\circ} \mathrm{C}\right): \delta_{\text {н }} 6.43(\mathrm{br} \mathrm{d}, J 7.2 \mathrm{~Hz}$, $1 \mathrm{H}), 5.10(\mathrm{~s}, 1 \mathrm{H}), 4.38(\mathrm{~s}, 1 \mathrm{H}), 4.19(\mathrm{~m}, 7 \mathrm{H}), 3.80(\mathrm{~m}, 1 \mathrm{H}), 3.23(\mathrm{td}, J 5.6,5.6,2.8 \mathrm{~Hz}, 1 \mathrm{H}), 2.94$ $(\mathrm{td}, J 5.6,5.6,2.8 \mathrm{~Hz}, 1 \mathrm{H}), 2.87$ (ddd, $J 14.6,5.6,2.8 \mathrm{~Hz}, 1 \mathrm{H}), 1.96(\mathrm{~m}, 2 \mathrm{H}), 1.66(\mathrm{~m}, 2 \mathrm{H}), 1.38$ $(\mathrm{m}, 2 \mathrm{H}), 1.21(\mathrm{~m}, 3 \mathrm{H}) ;{ }^{13} \mathrm{C} \mathrm{NMR}\left(\mathrm{CDCl}_{3}, 100 \mathrm{MHz}, 30^{\circ} \mathrm{C}\right): \delta_{\mathrm{c}} 167.3$ (IV), 166.9 (IV), 82.8 (IV), 69.1 (5C), 58.6, 68.3, 67.8, 55.9, 48.6, 38.8, 36.7, 33.0, 32.9, 28.2; HRMS m/z 395.1422 [M $\mathrm{C}_{21} \mathrm{H}_{27} \mathrm{~N}_{2} \mathrm{O}_{2} \mathrm{Fe}$ requires 395.1422]; Anal. Calcd for $\mathrm{C}_{21} \mathrm{H}_{27} \mathrm{~N}_{2} \mathrm{O}_{2} \mathrm{Fe}$ : C, 63.81; H, 6.88; N, 7.09. Found: C, 63.70; H, 6.61; N, 7.05.

N-Cyclohexyl-2-(2-oxoazetidin-1-yl)-2-(quinolin-4-yl)acetamide (1.27). White crystalline solid; Yield 69\%; mp 197-199 ${ }^{\circ} \mathrm{C}$ (EtOAc/hexane); IR (KBr, $v_{\max }, \mathrm{cm}^{-1}$ ) 3280s, 2928s, 1747vs, 1650vs, 766s; ${ }^{1} \mathrm{H}$ NMR $\left(\mathrm{CDCl}_{3}, 400 \mathrm{MHz}, 30{ }^{\circ} \mathrm{C}\right): \delta_{\text {н }} 8.93(\mathrm{~d}, J 4.4 \mathrm{~Hz}, 1 \mathrm{H}), 8.19$ (d, $J 8.3 \mathrm{~Hz}$, $1 \mathrm{H}), 8.12(\mathrm{~d}, J 7.8 \mathrm{~Hz}, 1 \mathrm{H}), 7.80(\mathrm{t}, J 7.5 \mathrm{~Hz}, 1 \mathrm{H}), 7.69$ (t, J 7.8Hz, 1H), 7.49 (d, J 4.4Hz, 1H), $6.06(\mathrm{~s}, 1 \mathrm{H}), 5.89(\mathrm{~d}, J 8.7 \mathrm{~Hz}, 1 \mathrm{H}), 3.90(\mathrm{~m}, 1 \mathrm{H}), 3.68$ (td, $J 5.6,5.6,2.6 \mathrm{~Hz}, 1 \mathrm{H}), 3.06$ (ddd, $J$ $14.1,5.4,2.4 \mathrm{~Hz}, 1 \mathrm{H}), 2.920(\mathrm{td}, J 5.6,5.6,2.6 \mathrm{~Hz}, 1 \mathrm{H}), 2.85$ (ddd, $J 14.1,5.4,2.4 \mathrm{~Hz}, 1 \mathrm{H}), 1.93$ $(\mathrm{m}, 2 \mathrm{H}), 1.70(\mathrm{~m}, 3 \mathrm{H}), 1.39(\mathrm{~m}, 2 \mathrm{H}), 1.19(\mathrm{~m}, 3 \mathrm{H}) ;{ }^{13} \mathrm{C} \mathrm{NMR}\left(\mathrm{CDCl}_{3}, 100 \mathrm{MHz}, 30{ }^{\circ} \mathrm{C}\right): \delta_{\mathrm{c}}$ 167.8 (IV), 166.9 (IV), 150.1, 148.7, 139.8, 130.5, 129.8, 127.7, 126.1 (IV), 122.8, 120.5, 55.0, 48.9, 39.0, 36.4, 32.7, 32.6, 25.3, 24.7, 24.6; HRMS $m / z 337.1793\left[\mathrm{M}^{+}, \mathrm{C}_{20} \mathrm{H}_{23} \mathrm{~N}_{3} \mathrm{O}_{2}\right.$ requires 337.1790]; Anal. Calcd for $\mathrm{C}_{20} \mathrm{H}_{23} \mathrm{~N}_{3} \mathrm{O}_{2}$ : C, 71.19; H, 6.87; N, 12.45. Found: C, 71.37; H, 6.89; $\mathrm{N}, 12.65$.

General procedure for Mannich Reactions. The terminal acetylene (1.0 equiv), aqueous formaldehyde (5.0 equiv), amine (1.2 eq) and copper(I) iodide (0.02 equiv) were stirred in commercial DMSO at $25{ }^{\circ} \mathrm{C}$ for $2 \mathrm{~h} . \mathrm{H}_{2} \mathrm{O}$ was added to the reaction mixture, which was basified to $\mathrm{pH}$ 8. The mixture was extracted with EtOAc, washed with brine, dried $\left(\mathrm{Na}_{2} \mathrm{SO}_{4}\right)$ and solvent evaporated to yield the crude products. 
N-Cyclohexyl-2-[3-(\{4-[benzyl(methyl)amino]but-2-ynyl\}oxy)phenyl]-2-(2-oxoazetidin-1yl)acetamide (2.1). Crude residue was purified via column chromatography, eluting with $\mathrm{MeOH} / \mathrm{DCM}(0.5: 9.5)$ to yield the product as a white powder, $(75.1 \mathrm{mg}, 72 \%) ; \mathrm{mp} 88-91{ }^{\circ} \mathrm{C}$; $\mathrm{R}_{\mathrm{f}}(\mathrm{MeOH} / \mathrm{DCM}, 0.5: 9.5)$ 0.33; IR (DCM, $\left.v_{\max }, \mathrm{cm}^{-1}\right) 2312 \mathrm{w}, 1742 \mathrm{~s}, 1672 \mathrm{~s}, 1249 \mathrm{~s}$ and $1025 \mathrm{~m} ;{ }^{1} \mathrm{H}$ NMR $\left(\mathrm{CDCl}_{3}, 300 \mathrm{MHz}, 30{ }^{\circ} \mathrm{C}\right): \delta_{\mathrm{H}} 7.26(\mathrm{~m}, 6 \mathrm{H}), 6.98(\mathrm{~m}, 3 \mathrm{H}), 5.90(\mathrm{~d}, J 7.5 \mathrm{~Hz}, 1 \mathrm{H}), 5.24(\mathrm{~s}$, $1 \mathrm{H}), 4.76(\mathrm{t}, J 1.8 \mathrm{~Hz}, 2 \mathrm{H}), 3.73(\mathrm{~m}, 1 \mathrm{H}), 3.56(\mathrm{td}, J 5.7,5.7,2.7 \mathrm{~Hz}, 1 \mathrm{H}), 3.50(\mathrm{~s}, 2 \mathrm{H}), 3.31(\mathrm{t}, \mathrm{J}$ $1.8 \mathrm{~Hz}, 2 \mathrm{H}, \mathrm{H} 7), 3.11$ (td, $J 5.7,5.7,2.7 \mathrm{~Hz}, 1 \mathrm{H}), 2.95$ (ddd, $J 14.7,5.7,2.7 \mathrm{~Hz}, 1 \mathrm{H}), 2.79$ (ddd, $J$

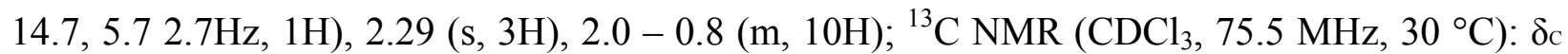
167.9 (IV), 167.4 (IV), 158.1 (IV), 138.1 (IV), 136.4 (IV), 130.0, 129.1 (2C), 128.3 (2C), 127.1, 121.0, 115.1, 114.9, 82.8 (IV), 80.0 (IV), 60.1, 59.9, 56.2, 48.6, 45.1, 41.9, 39.0, 36.2, 32.7, 29.6, 25.4, 24.7 and 24.6; HRMS $m / z 473.6162\left(\mathrm{M}^{+}, \mathrm{C}_{29} \mathrm{H}_{35} \mathrm{~N}_{3} \mathrm{O}_{3}\right.$ requires 473.6152); Anal. Calcd for $\mathrm{C}_{29} \mathrm{H}_{35} \mathrm{~N}_{3} \mathrm{O}_{3}$ : C, 73.54; H, 7.45; N, 8.87. Found: C, 73.24; H, 7.57; N, 8.80.

$\mathrm{N}$-Cyclohexyl-2-(3-\{[4-(diethylamino)but-2-ynyl]oxy\}phenyl)-2-(2-oxoazetidin-1-yl)-

acetamide (2.2). Crude residue was purified via column chromatography, eluting with $\mathrm{MeOH} /$ EtOAc (0.1:9.9 to 1:9) to yield the product as a white powder, (83.0 $\mathrm{mg}, 89 \%$ ); mp $102-$ $105^{\circ} \mathrm{C}$; $\mathrm{R}_{\mathrm{f}}$ (EtOAc) 0.12; IR (DCM, $\left.v_{\max }, \mathrm{cm}^{-1}\right) 2305 \mathrm{w}, 1741 \mathrm{~s}, 1672 \mathrm{~s}, 1248 \mathrm{~s}$ and $1022 \mathrm{~m} ;{ }^{1} \mathrm{H}$ NMR $\left(\mathrm{CDCl}_{3}, 300 \mathrm{MHz}, 30{ }^{\circ} \mathrm{C}\right): \delta_{\mathrm{H}} 7.29(\mathrm{~m}, 1 \mathrm{H}), 6.96(\mathrm{~m}, 3 \mathrm{H}), 5.94(\mathrm{~d}, J 7.8 \mathrm{~Hz}, 1 \mathrm{H}), 5.25(\mathrm{~s}, 1 \mathrm{H})$, $4.71(\mathrm{t}, J 2.1 \mathrm{~Hz}, 2 \mathrm{H}), 3.76(\mathrm{~m}, 1 \mathrm{H}), 3.60(\mathrm{td}, J 6.0,6.0,2.4 \mathrm{~Hz}, 1 \mathrm{H}), 3.44(\mathrm{t}, J 2.1 \mathrm{~Hz}, 2 \mathrm{H}), 3.17$ $(\mathrm{td}, J 6.0,6.0,2.4 \mathrm{~Hz}, 1 \mathrm{H}), 2.99(\mathrm{ddd}, J 14.7,6.0,2.4 \mathrm{~Hz}, 1 \mathrm{H}), 2.86$ (ddd, $J 14.7,6.0,2.4 \mathrm{~Hz}, 1 \mathrm{H})$, $2.51(\mathrm{q}, J 7.5 \mathrm{~Hz}, 4 \mathrm{H}), 2.0-1.1(\mathrm{~m}, 10 \mathrm{H}), 1.03(\mathrm{t}, J 7.5 \mathrm{~Hz}, 6 \mathrm{H}) ;{ }^{13} \mathrm{C} \mathrm{NMR}\left(\mathrm{CDCl}_{3}, 100 \mathrm{MHz}, 30\right.$ $\left.{ }^{\circ} \mathrm{C}\right): \delta_{\mathrm{c}} 167.9$ (IV), 167.4 (IV), 158.2 (IV), 136.3 (IV), 130.0, 120.9, 115.1, 114.8, 82.9 (IV), 79.2 (IV), 60.1, 56.3, 48.7, 47.2 (2C), 41.0, 39.0, 36.3, 32.8, 32.7, 25.4, 24.7, 24.6 and 12.5 (2C); HRMS $m / z 425.5715\left(\mathrm{M}^{+} \mathrm{C}_{25} \mathrm{H}_{35} \mathrm{~N}_{3} \mathrm{O}_{3}\right.$ requires $\mathrm{M}$, 425.5712); Anal. Calcd for $\mathrm{C}_{25} \mathrm{H}_{35} \mathrm{~N}_{3} \mathrm{O}_{3}$ : $\mathrm{C}$, 70.56; H, 8.29; N, 9.87; Found: C, 70.41; H, 7.98; N, 9.85.

$\mathrm{N}$-Cyclohexyl-2-(2-oxoazetidin-1-yl)-2-\{3-[(4-pyrrolidin-1-ylbut-2-ynyl)oxy]phenyl\}

acetamide (2.3). Crude residue was purified via column chromatography, eluting with $\mathrm{MeOH} /$ EtOAc (0.3:9.7 to 1:9) to yield the product as a white powder, (74.2 $\mathrm{mg}, 80 \%)$; mp 89 $91{ }^{\circ} \mathrm{C} ; \mathrm{R}_{\mathrm{f}}(\mathrm{MeOH} / \mathrm{EtOAc}, 0.3: 9.7)$ 0.11; IR (DCM, $\left.v_{\max }, \mathrm{cm}^{-1}\right) 2305 \mathrm{w}, 1742 \mathrm{~s}, 1672 \mathrm{~s}, 1245 \mathrm{~s}$ and $1022 \mathrm{~m} ;{ }^{1} \mathrm{H} \mathrm{NMR}\left(\mathrm{CDCl}_{3}, 300 \mathrm{MHz}, 30{ }^{\circ} \mathrm{C}\right): \delta_{\mathrm{H}} 7.26(\mathrm{~m}, 1 \mathrm{H}), 6.97(\mathrm{~m}, 3 \mathrm{H}), 6.00(\mathrm{~d}, J 7.8 \mathrm{~Hz}$, $1 \mathrm{H}), 5.26(\mathrm{~s}, 1 \mathrm{H}), 4.71(\mathrm{t}, J 2.1 \mathrm{~Hz}, 2 \mathrm{H}), 3.76(\mathrm{~m}, 1 \mathrm{H}), 3.61(\mathrm{td}, J 6.0,6.0,2.4 \mathrm{~Hz}, 1 \mathrm{H}), 3.44(\mathrm{t}, J$ $2.1 \mathrm{~Hz}, 2 \mathrm{H}), 3.17(\mathrm{td}, J 6.0,6.0,2.4 \mathrm{~Hz}, 1 \mathrm{H}), 2.99$ (ddd, $J 14.7,6.0,2.4 \mathrm{~Hz}, 1 \mathrm{H}), 2.86$ (ddd, $J 14.7$, 6.0, 2.4Hz, 1H), $2.59(\mathrm{~m}, 4 \mathrm{H}), 1.78(\mathrm{~m}, 4 \mathrm{H}), 2.1-1.0(\mathrm{~m}, 10 \mathrm{H}) ;{ }^{13} \mathrm{C} \mathrm{NMR}\left(\mathrm{CDCl}_{3}, 100 \mathrm{MHz}, 30\right.$ $\left.{ }^{\circ} \mathrm{C}\right): \delta_{\mathrm{c}} 167.9$ (IV), 167.4 (IV), 158.2 (IV), 136.4 (IV), 130.0, 121.0, 115.1, 114.9, 83.7 (IV), 78.8 (IV), 60.0, 56.3, 52.6 (2C), 48.7, 43.3, 39.0, 36.3, 32.7, 32.6, 25.4, 24.7, 24.6 and 23.8 (2C); HRMS m/z $423.5558\left(\mathrm{M}^{+} \mathrm{C}_{25} \mathrm{H}_{33} \mathrm{~N}_{3} \mathrm{O}_{3}\right.$ requires 423.5553); Anal. Calcd for $\mathrm{C}_{25} \mathrm{H}_{33} \mathrm{~N}_{3} \mathrm{O}_{3}$ : C, 70.89; H, 7.85; N, 9.92\%. Found: C, 70.67; H, 7.81; N, 9.75.

$\mathrm{N}$-Cyclohexyl-2-(2-oxoazetidin-1-yl)-2-\{3-[(4-piperidin-1-ylbut-2-ynyl)oxy]phenyl\}

acetamide (2.4). Crude residue was purified via column chromatography, eluting with $\mathrm{MeOH} / \mathrm{EtOAc}(0.3: 9.7)$ to yield the product as a white powder, (70.8 mg, 73\%); mp $107-109$ ${ }^{\circ} \mathrm{C} ; \mathrm{R}_{\mathrm{f}}(\mathrm{MeOH} / \mathrm{EtOAc}, 0.3: 9.7)$ 0.24; IR (DCM, $\left.v_{\max }, \mathrm{cm}^{-1}\right) 2305 \mathrm{w}, 1741 \mathrm{~s}, 1672 \mathrm{~s}, 1262 \mathrm{~s}$ and 
$1015 \mathrm{~m} ;{ }^{1} \mathrm{H} \mathrm{NMR}\left(\mathrm{CDCl}_{3}, 300 \mathrm{MHz}, 30{ }^{\circ} \mathrm{C}\right): \delta_{\mathrm{H}} 7.29(\mathrm{~m}, 1 \mathrm{H}), 6.96(\mathrm{~m}, 3 \mathrm{H}), 5.92(\mathrm{~d}, J 7.8 \mathrm{~Hz}$, $1 \mathrm{H}), 5.25(\mathrm{~s}, 1 \mathrm{H}), 4.71(\mathrm{t}, J 1.8 \mathrm{~Hz}, 2 \mathrm{H}), 3.78(\mathrm{~m}, 1 \mathrm{H}), 3.60(\mathrm{td}, J 5.7,5.7,2.4 \mathrm{~Hz}, 1 \mathrm{H}), 3.28(\mathrm{t}, J$ $1.8 \mathrm{~Hz}, 2 \mathrm{H}), 3.17(\mathrm{td}, J 5.7,5.7,2.4 \mathrm{~Hz}, 1 \mathrm{H}), 3.01(\mathrm{ddd}, J 14.7,5.7,2.4 \mathrm{~Hz}, 1 \mathrm{H}), 2.87$ (ddd, $J 14.7$, 5.7, 2.4Hz, 1H), $2.45(\mathrm{~m}, 4 \mathrm{H}), 1.9-1.0(\mathrm{~m}, 16 \mathrm{H}) ;{ }^{13} \mathrm{C} \mathrm{NMR}\left(\mathrm{CDCl}_{3}, 100 \mathrm{MHz}, 30{ }^{\circ} \mathrm{C}\right): \delta_{\mathrm{c}} 167.9$ (IV), 167.4 (IV), 158.2 (IV), 136.3 (IV), 130.1, 121.0, 115.1, 114.9, 83.5 (IV), 79.3 (IV), 60.1, 56.4, 53.4 (2C), 48.7, 47.9, 39.0, 36.3, 32.8, 32.7, 25.9 (2C), 25.4, 24.7, 24.6 and 23.8; HRMS $m / z 437.5827\left(\mathrm{M}^{+} \mathrm{C}_{26} \mathrm{H}_{35} \mathrm{~N}_{3} \mathrm{O}_{3}\right.$ requires 437.5822); Anal. Calcd for $\mathrm{C}_{26} \mathrm{H}_{35} \mathrm{~N}_{3} \mathrm{O}_{3}$ : C, 71.37; $\mathrm{H}$, 8.06; N, 9.60\%. Found: C, 71.61; H, 8.14; N, 9.61 .

\section{$\mathrm{N}$-Cyclohexyl-2-(2-oxoazetidin-1-yl)-2-(3-\{[4-(4-isopropylpiperazin-1-yl)but-2-ynyl]oxy\}}

phenyl)acetamide (2.5). Crude residue was purified via column chromatography, eluting with $\mathrm{MeOH} / \mathrm{DCM}(0.5: 9.5)$ to yield the product as a light yellow oil, $(91.0 \mathrm{mg}, 86 \%)$; $\mathrm{R}_{\mathrm{f}}$ (MeOH/DCM, 0.5:9.5) 0.26; IR (DCM, $v_{\max }, \mathrm{cm}^{-1}$ ) 2305w, 1740s, 1675s, 1265s and 1022m; ${ }^{1} \mathrm{H}$ NMR $\left(\mathrm{CDCl}_{3}, 400 \mathrm{MHz}, 30{ }^{\circ} \mathrm{C}\right): \delta_{\mathrm{H}} 7.26(\mathrm{~m}, 1 \mathrm{H}), 6.93(\mathrm{~m}, 3 \mathrm{H}), 6.06(\mathrm{br} \mathrm{s}, 1 \mathrm{H}), 5.25(\mathrm{~s}, 1 \mathrm{H})$, $4.67(\mathrm{t}, J 2.0 \mathrm{~Hz}, 2 \mathrm{H}), 3.73(\mathrm{~m}, 1 \mathrm{H}), 3.59(\mathrm{td}, J 5.6,5.6,2.8 \mathrm{~Hz}, 1 \mathrm{H}), 3.29(\mathrm{t}, J 2.0 \mathrm{~Hz}, 2 \mathrm{H}), 3.16$ (td, $J 5.6,5.6,2.8 \mathrm{~Hz}, 1 \mathrm{H}), 2.96$ (ddd, $J 14.8,5.6,2.8 \mathrm{~Hz}, 1 \mathrm{H}), 2.83$ (ddd, $J 14.8,5.6,2.8 \mathrm{~Hz}, 1 \mathrm{H}$ ), 2.65 (septet, $J 6.4 \mathrm{~Hz}, 1 \mathrm{H}), 2.56$ (br s, $8 \mathrm{H}), 2.0-1.1(\mathrm{~m}, 10 \mathrm{H}), 1.02(\mathrm{~d}, J 6.4 \mathrm{~Hz}, 6 \mathrm{H}) ;{ }^{13} \mathrm{C}$ NMR $\left(\mathrm{CDCl}_{3}, 100 \mathrm{MHz}, 30{ }^{\circ} \mathrm{C}\right): \delta_{\mathrm{c}} 167.8$ (IV), 167.4 (IV), 158.2 (IV), 136.4 (IV), 130.1, 120.9, 115.1, 115.0, 83.0 (IV), 79.7 (IV), 59.9, 56.3 (2C), 54.5, 52.3, 48.7, 48.4 (2C), 47.1, 39.0, 36.3 (2C), 32.8 (2C), 25.4 (2C), 24.7, 18.5; m/z $480.6514\left(\mathrm{M}^{+} \mathrm{C}_{28} \mathrm{H}_{40} \mathrm{~N}_{4} \mathrm{O}_{3}\right.$ requires 480.6506); Anal. Calcd for $\mathrm{C}_{28} \mathrm{H}_{40} \mathrm{~N}_{4} \mathrm{O}_{3}$ : C, 69.97; H, 8.39; N, 11.66. Found: C, 70.14; H, 8.20; N, 11.60.

\section{$\mathrm{N}$-Cyclohexyl-2-(2-oxoazetidin-1-yl)-2-\{3-[(4-piperazin-1-ylbut-2-ynyl)oxy]phenyl\}}

acetamide (2.6). Crude residue was purified via column chromatography, eluting with $\mathrm{MeOH} /$ EtOAc $(0.5: 9.5)$ to yield the product as a white powder, $(67.4 \mathrm{mg}, 65 \%)$; mp $115-117$ ${ }^{\circ} \mathrm{C} ; \mathrm{R}_{\mathrm{f}}\left(\mathrm{MeOH} /\right.$ EtOAc, 0.3:9.7) 0.14; IR (DCM, v $\left.v_{\max }, \mathrm{cm}^{-1}\right) 2305 \mathrm{w}, 1741 \mathrm{~s}, 1672 \mathrm{~s}, 1265 \mathrm{~s}$ and $1025 \mathrm{~m} ;{ }^{1} \mathrm{H} \mathrm{NMR}\left(\mathrm{CDCl}_{3}, 400 \mathrm{MHz}, 30{ }^{\circ} \mathrm{C}\right): \delta_{\mathrm{H}} 7.25(\mathrm{~m}, 1 \mathrm{H}), 6.92(\mathrm{~m}, 3 \mathrm{H}), 6.18(\mathrm{~d}, J 8.0 \mathrm{~Hz}$, $1 \mathrm{H}), 5.29(\mathrm{~s}, 1 \mathrm{H}), 4.67(\mathrm{t}, J 1.6 \mathrm{~Hz}, 2 \mathrm{H}), 3.73(\mathrm{~m}, 1 \mathrm{H}), 3.59(\mathrm{td}, J 5.6,5.6,2.8 \mathrm{~Hz}, 1 \mathrm{H}), 3.31(\mathrm{t}, J$ $1.6 \mathrm{~Hz}, 2 \mathrm{H}), 3.17(\mathrm{td}, J 5.6,5.6,2.8 \mathrm{~Hz}, 1 \mathrm{H}), 2.95(\mathrm{ddd}, J 14.8,5.6,2.8 \mathrm{~Hz}, 1 \mathrm{H}), 2.83$ (ddd, $J 14.8$, $5.6,2.8 \mathrm{~Hz}, 1 \mathrm{H}), 2.56$ (br s, 4H), 2.17 (br s, $2 \mathrm{H}), 1.84$ (br s, $4 \mathrm{H}), 1.7-1.1(\mathrm{~m}, 8 \mathrm{H}) ;{ }^{13} \mathrm{C} \mathrm{NMR}$ $\left(\mathrm{CDCl}_{3}, 100 \mathrm{MHz}, 30{ }^{\circ} \mathrm{C}\right): \delta_{\mathrm{c}} 167.9$ (IV), 167.4 (IV), 158.1 (IV), 136.4 (IV), 130.1, 120.9, 115.0, 114.9, 82.7 (IV), 80.0 (IV), 59.8, 59.7, 56.3, 51.7 (2C), 48.7, 47.0, 39.1 (2C), 36.3, 32.8 (2C), 25.5, 24.8, 24.7; HRMS $m / z 438.5704\left(\mathrm{M}^{+} \mathrm{C}_{25} \mathrm{H}_{34} \mathrm{~N}_{4} \mathrm{O}_{3}\right.$ requires 438.5700); Anal. Calcd for $\mathrm{C}_{25} \mathrm{H}_{34} \mathrm{~N}_{4} \mathrm{O}_{3}$ : C, 68.47; H, 7.81; N, 12.77. Found: C, 68.20; H, 7.53; N, 12.59 .

\section{$\mathrm{N}$-Cyclohexyl-2-\{3-[(4-morpholin-4-ylbut-2-ynyl)oxy]phenyl\}-2-(2-oxoazetidin-1-yl)}

acetamide (2.7). Crude residue was purified via column chromatography, eluting with $\mathrm{MeOH} /$ EtOAc (0.1:9.9 to 1:9) to yield the product as a white powder, $(74.2 \mathrm{mg}, 77 \%)$; mp $102-$ $105^{\circ} \mathrm{C}$; $\mathrm{R}_{\mathrm{f}}$ (EtOAc) 0.17; IR (DCM, $\left.v_{\max }, \mathrm{cm}^{-1}\right) 2305 \mathrm{w}, 1740 \mathrm{~s}, 1682 \mathrm{~s}, 1263 \mathrm{~s}$ and $1031 \mathrm{~m} ;{ }^{1} \mathrm{H} \mathrm{NMR}$ $\left(\mathrm{CDCl}_{3}, 300 \mathrm{MHz}, 30^{\circ} \mathrm{C}\right): \delta_{\mathrm{H}} 7.29(\mathrm{~m}, 1 \mathrm{H}), 6.96(\mathrm{~m}, 3 \mathrm{H}), 6.18(\mathrm{~d}, J 7.5 \mathrm{~Hz}, 1 \mathrm{H}), 5.29(\mathrm{~s}, 1 \mathrm{H})$, $4.72(\mathrm{t}, J 1.8 \mathrm{~Hz}, 2 \mathrm{H}), 3.71(\mathrm{~m}, 4 \mathrm{H}), 3.61(\mathrm{td}, J 5.4,5.4,2.4 \mathrm{~Hz}, 1 \mathrm{H}), 3.33(\mathrm{t}, J 1.8 \mathrm{~Hz}, 2 \mathrm{H}), 3.15$ (td, $J 5.4,5.4,2.4 \mathrm{~Hz}, 1 \mathrm{H}), 2.99$ (ddd, $J 14.7,5.4,2.4 \mathrm{~Hz}, 1 \mathrm{H}), 2.85$ (ddd, $J 14.7,5.4,2.4 \mathrm{~Hz}, 1 \mathrm{H}$ ), $4.50(\mathrm{~m}, 4 \mathrm{H}), 1.9-1.0(\mathrm{~m}, 10 \mathrm{H}) ;{ }^{13} \mathrm{C} \mathrm{NMR}\left(\mathrm{CDCl}_{3}, 100 \mathrm{MHz}, 30{ }^{\circ} \mathrm{C}\right): \delta_{\mathrm{c}} 167.8$ (IV), 167.4 (IV), 
158.1 (IV), 136.4 (IV), 130.0, 120.9, 115.4, 114.9, 82.5 (IV), 80.2 (IV), 66.8 (2C), 59.7, 56.2, 52.1 (2C), 48.7, 47.4, 38.9, 36.3, 32.8, 32.7, 25.5, 24.8 and 24.7; HRMS m/z 439.5552 $\left(\mathrm{M}^{+}\right.$ $\mathrm{C}_{25} \mathrm{H}_{33} \mathrm{~N}_{3} \mathrm{O}_{4}$ requires 439.5547); Anal. Calcd for $\mathrm{C}_{25} \mathrm{H}_{33} \mathrm{~N}_{3} \mathrm{O}_{4}$ : C, 68.31; H, 7.57; N, 9.56. Found: C, 68.45; H, 7.67; N, 9.40.

3-[(4-Morpholin-4-ylbut-2-ynyl)oxy]benzaldehyde (7). Crude residue was purified via column chromatography, eluting with $\mathrm{MeOH} / \mathrm{EtOAc}(0.3: 9.7)$ to yield the product as a light-yellow oil, (1.57 g, 88\%); $\mathrm{R}_{\mathrm{f}}$ (hexane/EtOAc, 2:8) 0.26; IR (DCM, $\left.v_{\max }, \mathrm{cm}^{-1}\right) 3058 \mathrm{w}, 2862 \mathrm{w}, 2812 \mathrm{w}, 2312 \mathrm{w}$, 1700s, $1586 \mathrm{~m}, 1478 \mathrm{~m}$ and 1266s; ${ }^{1} \mathrm{H} \mathrm{NMR}\left(\mathrm{CDCl}_{3}, 300 \mathrm{MHz}, 30{ }^{\circ} \mathrm{C}\right): \delta_{\mathrm{H}} 9.97(\mathrm{~s}, 1 \mathrm{H}), 7.48(\mathrm{~m}$, $3 \mathrm{H}), 7.23(\mathrm{~m}, 1 \mathrm{H}), 4.78(\mathrm{t}, J 2.0 \mathrm{~Hz}, 2 \mathrm{H}), 3.69(\mathrm{~m}, 4 \mathrm{H}), 3.31(\mathrm{t}, J 2.0 \mathrm{~Hz}, 2 \mathrm{H}), 2.49(\mathrm{~m}, 4 \mathrm{H}) ;{ }^{13} \mathrm{C}$ NMR $\left(\mathrm{CDCl}_{3}, 100 \mathrm{MHz}, 30{ }^{\circ} \mathrm{C}\right.$ ): $\delta_{\mathrm{c}} 191.8$ (IV), 158.2 (IV), 137.8 (IV), 130.1, 124.1, 122.3, 113.5, 83.1 (IV), 79.6 (IV), 66.8 (2C), 56.3, 52.3 (2C) and 47.4; HRMS m/z 259.3053 (M $\mathrm{C}_{15} \mathrm{H}_{17} \mathrm{NO}_{3}$ requires 259.3049); Anal. Calcd for $\mathrm{C}_{15} \mathrm{H}_{17} \mathrm{NO}_{3}$ : C, 69.48; H, 6.61; N, 5.40. Found: C, 69.40; H, 6.30; N, 5.68.

General synthesis for $\boldsymbol{\beta}$-lactams. $\beta$-alanine (1.1 equiv), cyclohexyl isocyanide (1.0 equiv) and the appropriate aldehyde (1.0 equiv) were added to $\mathrm{MeOH}$ and allowed to stir at $25{ }^{\circ} \mathrm{C}$ for $48-72$ hours. The reaction was monitored by TLC (1:1 EtOAc/hexane). Once the aldehyde spot had disappeared the reaction was deemed complete. The solvent was removed from the reaction mixture under reduced pressure. The crude mixture was purified using silica gel chromatography eluting with 1:1 EtOAc/hexane. This method is a slight variation on the method described by Pitlik and Townsend. ${ }^{9}$

$\mathrm{N}$-(4-Methoxyphenyl)-2-\{3-[(4-morpholin-4-ylbut-2-ynyl)oxy]phenyl\}-2-(2-oxoazetidin-1-

yl)acetamide (3.1). Crude residue was purified via column chromatography, eluting with EtOAc to yield the product as a light-brown oil, (0.172 $\mathrm{g}, 97 \%)$; (EtOAc) 0.22; IR (DCM, $v_{\max }, \mathrm{cm}^{-1}$ ) 2304w, 1743s, 1282s, 1262s and $1031 \mathrm{~m} ;{ }^{1} \mathrm{H} \mathrm{NMR}\left(\mathrm{CDCl}_{3}, 400 \mathrm{MHz}, 30{ }^{\circ} \mathrm{C}\right): \delta_{\mathrm{H}} 7.39(\mathrm{~d}, J$ $8.8 \mathrm{~Hz}, 2 \mathrm{H}), 7.27(\mathrm{~m}, 1 \mathrm{H}), 7.04(\mathrm{~m}, 2 \mathrm{H}), 6.93(\mathrm{~m}, 1 \mathrm{H}), 6.80(\mathrm{~d}, J 8.8 \mathrm{~Hz}, 2 \mathrm{H}), 5.51(\mathrm{~s}, 1 \mathrm{H}), 5.26$ $(\mathrm{s}, 1 \mathrm{H}), 4.70(\mathrm{~s}, 2 \mathrm{H}), 3.74(\mathrm{~s}, 3 \mathrm{H}), 3.67(\mathrm{~m}, 5 \mathrm{H}), 3.28(\mathrm{~s}, 2 \mathrm{H}), 3.19(\mathrm{td}, J 5.6,5.6,2.8 \mathrm{~Hz}, 1 \mathrm{H})$, 2.99 (ddd, $J 14.8,5.6,2.8 \mathrm{~Hz}, 1 \mathrm{H}), 2.85(\mathrm{ddd}, J 14.8,5.6,2.8 \mathrm{~Hz}, 1 \mathrm{H}), 2.45(\mathrm{~m}, 4 \mathrm{H}) ;{ }^{13} \mathrm{C}$ NMR $\left(\mathrm{CDCl}_{3}, 100 \mathrm{MHz}, 30{ }^{\circ} \mathrm{C}\right): \delta_{\mathrm{c}} 168.1$ (IV), 166.5 (IV), 158.1 (IV), 156.6 (IV), 135.9 (IV), 130.8 (IV), 130.2, 121.7 (2C), 120.9, 115.9, 114.8, 114.1 (2C), 82.6 (IV), 80.3 (IV), 66.8 (2C), 60.2, 56.2, 55.5, 51.9 (2C), 47.3, 39.2 and 36.3; HRMS $m / z 463.5340\left(\mathrm{M}^{+} \mathrm{C}_{26} \mathrm{H}_{29} \mathrm{~N}_{3} \mathrm{O}_{5}\right.$ requires 463.5334); Anal. Calcd for $\mathrm{C}_{26} \mathrm{H}_{29} \mathrm{~N}_{3} \mathrm{O}_{5}$ : C, 67.37; H, 6.31; N, 9.07. Found: C, 67.41; H, 6.59; N, 9.38 .

N-(1,1,3,3-Tetramethylbutyl)-2-\{3-[(4-morpholin-4-ylbut-2-ynyl)oxy]phenyl\}-2-(2oxoazetidin-1-yl)acetamide (3.2). Crude residue was purified via column chromatography, eluting with $\mathrm{MeOH} / \mathrm{EtOAc}(0.1: 9.9)$ to yield the product as a white powder, $(0.110 \mathrm{~g}, 62 \%)$; $\mathrm{mp}$ $86-89{ }^{\circ} \mathrm{C}$; $\mathrm{R}_{\mathrm{f}}\left(\right.$ EtOAc) 0.38; IR (DCM, $\left.v_{\max }, \mathrm{cm}^{-1}\right) 2305 \mathrm{w}, 1737 \mathrm{~s}, 1694 \mathrm{~s}, 1266 \mathrm{~s}$ and $1035 \mathrm{~m} ;{ }^{1} \mathrm{H}$ NMR $\left(\mathrm{CDCl}_{3}, 400 \mathrm{MHz}, 30^{\circ} \mathrm{C}\right): \delta_{\mathrm{H}} 7.27(\mathrm{~m}, 1 \mathrm{H}), 6.93(\mathrm{~m}, 3 \mathrm{H}), 5.93(\mathrm{~s}, 1 \mathrm{H}), 5.19(\mathrm{~s}, 1 \mathrm{H}), 4.69$ $(\mathrm{s}, 2 \mathrm{H}), 3.69(\mathrm{~m}, 4 \mathrm{H}), 3.59(\mathrm{td}, J 6.0,6.0,2.8 \mathrm{~Hz}, 1 \mathrm{H}), 3.30(\mathrm{~s}, 2 \mathrm{H}), 3.14(\mathrm{td}, J 6.0,6.0,2.8 \mathrm{~Hz}$, 1H), 2.95 (ddd, $J 14.8,6.0,2.8 \mathrm{~Hz}, 1 \mathrm{H}), 2.84$ (ddd, $J 14.8,6.0,2.8 \mathrm{~Hz}, 1 \mathrm{H}), 2.49$ (m, $4 \mathrm{H}), 1.74$ (d, 
$J 14.8 \mathrm{~Hz}, 1 \mathrm{H}), 1.56(\mathrm{~d}, J 14.8 \mathrm{~Hz}, 1 \mathrm{H}), 1.38(\mathrm{~s}, 3 \mathrm{H}), 1.34(\mathrm{~s}, 3 \mathrm{H}), 0.89(\mathrm{~s}, 9 \mathrm{H}) ;{ }^{13} \mathrm{C} \mathrm{NMR}\left(\mathrm{CDCl}_{3}\right.$, $100 \mathrm{MHz}, 30{ }^{\circ} \mathrm{C}$ ): $\delta_{\mathrm{c}} 168.1$ (IV), 167.2 (IV), 155.6 (IV), 136.8 (IV), 130.1, 121.1, 115.2, 115.0, 82.5 (IV), 80.1 (IV), 66.8 (2C), 60.4, 56.2, 52.1 (2C), 52.0, 47.4, 39.0, 36.3, 31.6, 31.5, 31.4, 29.0 and 28.7; HRMS $m / z 469.6256\left(\mathrm{M}^{+} \mathrm{C}_{27} \mathrm{H}_{39} \mathrm{~N}_{3} \mathrm{O}_{4}\right.$ requires 469.6244); Anal. Calcd for $\mathrm{C}_{27} \mathrm{H}_{39} \mathrm{~N}_{3} \mathrm{O}_{4}$ : C, 69.05; H, 8.37; N, 8.95. Found: C, 69.13; H, 8.54; N, 9.06.

$\mathrm{N}$-(1-Methylbutyl)-2-\{3-[(4-morpholin-4-ylbut-2-ynyl)oxy]phenyl\}-2-(2-oxoazetidin-1-

yl)acetamide (3.3). Crude residue was purified via column chromatography, eluting with $\mathrm{MeOH} /$ EtOAc (0.1:9.9) to yield the product as a colourless oil, $(0.148 \mathrm{~g}, 91 \%)$; $\mathrm{R}_{\mathrm{f}}$ (EtOAc) 0.33; IR (DCM, $\left.v_{\max }, \mathrm{cm}^{-1}\right) 2305 \mathrm{w}, 1735 \mathrm{~s}, 1695 \mathrm{~s}, 1266 \mathrm{~s}$ and $1024 \mathrm{~m} ;{ }^{1} \mathrm{H} \mathrm{NMR}\left(\mathrm{CDCl}_{3}, 400 \mathrm{MHz}, 30\right.$ $\left.{ }^{\circ} \mathrm{C}\right): \delta_{\mathrm{H}} 7.24(\mathrm{~m}, 1 \mathrm{H}), 6.95(\mathrm{~m}, 3 \mathrm{H}), 6.10(\mathrm{~m}, 1 \mathrm{H}), 5.29(\mathrm{~s}, 1 \mathrm{H}), 4.69(\mathrm{~s}, 2 \mathrm{H}), 3.95(\mathrm{~m}, 1 \mathrm{H}), 3.68$ $(\mathrm{m}, 4 \mathrm{H}), 3.60(\mathrm{td}, J 5.6,5.6,3.2 \mathrm{~Hz}, 1 \mathrm{H}), 3.30(\mathrm{~s}, 2 \mathrm{H}), 3.12(\mathrm{td}, J 5.6,5.6,3.2 \mathrm{~Hz}, 1 \mathrm{H}), 2.94$ (ddd, $J 14.8,5.6,3.2 \mathrm{~Hz}, 1 \mathrm{H}), 2.85(\mathrm{ddd}, J 14.8,5.6,3.2 \mathrm{~Hz}, 1 \mathrm{H}), 2.48(\mathrm{~m}, 4 \mathrm{H}), 1.4-0.7(\mathrm{~m}, 10 \mathrm{H}) ;{ }^{13} \mathrm{C}$ NMR $\left(\mathrm{CDCl}_{3}, 100 \mathrm{MHz}, 30{ }^{\circ} \mathrm{C}\right): \delta_{\mathrm{c}} 167.8$ (IV), 167.6 (IV), 158.1 (IV), 136.4 (IV), 130.1, 120.9, 115.4, 114.9, 82.5 (IV), 80.2 (IV), 66.8 (2C), 59.7, 56.2, 52.0 (2C), 47.4, 45.5, 38.9, 36.3, 27.3, 20.6, 19.2 and 13.9; HRMS $m / z 427.5444\left(\mathrm{M}^{+} \mathrm{C}_{24} \mathrm{H}_{33} \mathrm{~N}_{3} \mathrm{O}_{4}\right.$ requires 427.5437); Anal. Calcd for $\mathrm{C}_{24} \mathrm{H}_{33} \mathrm{~N}_{3} \mathrm{O}_{4}$ : C, 67.42; H, 7.78; N, 9.83. Found: C, 67.53; H, 7.96; N, 9.57.

\section{$\mathrm{N}$-2-Naphthyl-2-\{3-[(4-morpholin-4-ylbut-2-ynyl)oxy]phenyl\}-2-(2-oxoazetidin-1-}

yl)acetamide (3.4). Crude residue was purified via column chromatography, eluting with hexane/EtOAc (3:7) to EtOAc, to yield the product as a light-brown oil, $(0.152 \mathrm{~g}, 82 \%)$; $\mathrm{R}_{\mathrm{f}}$ (EtOAc) 0.26; IR (DCM, $\left.v_{\max }, \mathrm{cm}^{-1}\right) 2305 \mathrm{w}, 1737 \mathrm{~s}, 1694 \mathrm{~s}, 1266 \mathrm{~s}$ and $1021 \mathrm{~m} ;{ }^{1} \mathrm{H} \mathrm{NMR}\left(\mathrm{CDCl}_{3}\right.$, $\left.400 \mathrm{MHz}, 30{ }^{\circ} \mathrm{C}\right): \delta_{\mathrm{H}} 7.73(\mathrm{~m}, 3 \mathrm{H}), 7.4-7.2(\mathrm{~m}, 5 \mathrm{H}), 7.08(\mathrm{~m}, 2 \mathrm{H}), 6.95(\mathrm{~m}, 1 \mathrm{H}), 5.60(\mathrm{~s}, 1 \mathrm{H})$, $5.26(\mathrm{~s}, 1 \mathrm{H}), 4.71(\mathrm{~s}, 2 \mathrm{H}), 3.71(\mathrm{~m}, 5 \mathrm{H}), 3.25(\mathrm{~m}, 3 \mathrm{H}), 3.03$ (ddd, J 14.8, 5.6, 2.8Hz, 1H), 2.92 $(\mathrm{ddd}, J 14.8,5.6,2.8 \mathrm{~Hz}, 1 \mathrm{H}), 2.50-2.42(\mathrm{~m}, 4 \mathrm{H}) ;{ }^{13} \mathrm{C} \mathrm{NMR}\left(\mathrm{CDCl}_{3}, 100 \mathrm{MHz}, 30{ }^{\circ} \mathrm{C}\right): \delta_{\mathrm{c}} 168.3$ (IV), 167.0 (IV), 158.1 (IV), 135.7 (IV), 135.2 (IV), 133.8 (IV), 130.8 (IV), 130.2, 128.7, 127.7, $127.5,126.5,125.1,120.9,119.8,116.9,116.0,114.8,82.6$ (IV), 80.2 (IV), 66.8 (2C), 60.6, 56.2, 51.9 (2C), 47.3, 39.3 and 36.3; HRMS $m / z 483.5674\left(\mathrm{M}^{+} \mathrm{C}_{29} \mathrm{H}_{29} \mathrm{~N}_{3} \mathrm{O}_{4}\right.$ requires 483.5670); Anal. Calcd for $\mathrm{C}_{29} \mathrm{H}_{29} \mathrm{~N}_{3} \mathrm{O}_{4}$ : C, 72.03; H, 6.04; N, 8.69. Found: C, 72.33; H, 6.27; N, 8.77.

$\mathrm{N}$-\{[(4-Methoxyphenyl)sulfonyl]methyl\}-2-\{3-[(4-morpholin-4-ylbut-2-ynyl)oxy]phenyl\}-2(2-oxoazetidin-1-yl)acetamide (3.5). Crude residue was purified via column chromatography, eluting with $\mathrm{MeOH} / \mathrm{EtOAc}(0.1: 9.9$ to $0.3: 9.7)$, to yield the product as a light-brown oil, (0.143 $\mathrm{g}, 69 \%) ; \mathrm{R}_{\mathrm{f}}\left(\right.$ EtOAc) 0.16; IR (DCM, $v_{\max }, \mathrm{cm}^{-1}$ ) 2305w, 1745s, 1700s, 1324s, 1266s, 1145s and 1031; ${ }^{1} \mathrm{H} \mathrm{NMR}\left(\mathrm{CDCl}_{3}, 400 \mathrm{MHz}, 30^{\circ} \mathrm{C}\right): \delta_{\text {н }} 7.64(\mathrm{~m}, 3 \mathrm{H}), 7.25(\mathrm{~m}, 2 \mathrm{H}), 6.94(\mathrm{~m}, 1 \mathrm{H}), 6.89(\mathrm{~m}$, $1 \mathrm{H}), 6.77(\mathrm{~m}, 1 \mathrm{H}), 5.36(\mathrm{~s}, 1 \mathrm{H}), 4.74(\mathrm{dd}, J 14.0,6.8 \mathrm{~Hz}, 1 \mathrm{H}), 4.72(\mathrm{~s}, 2 \mathrm{H}), 4.51(\mathrm{dd}, J 14.0$, $6.8 \mathrm{~Hz}, 1 \mathrm{H}), 3.70(\mathrm{~m}, 4 \mathrm{H}), 3.41(\mathrm{td}, J 5.6,5.6,2.8 \mathrm{~Hz}, 1 \mathrm{H}), 3.34(\mathrm{~s}, 2 \mathrm{H}), 3.02(\mathrm{td}, J 5.6,5.6$, $2.8 \mathrm{~Hz}, 1 \mathrm{H}), 2.90$ (ddd, $J 14.4,5.6,2.8 \mathrm{~Hz}, 1 \mathrm{H}), 2.81$ (ddd, $J 14.4,5.6,2.8 \mathrm{~Hz}, 1 \mathrm{H}), 2.51$ (m, 4H), $2.41(\mathrm{~s}, 3 \mathrm{H}) ;{ }^{13} \mathrm{C} \mathrm{NMR}\left(\mathrm{CDCl}_{3}, 100 \mathrm{MHz}, 30{ }^{\circ} \mathrm{C}\right.$ ): $\delta_{\mathrm{c}} 168.3$ (IV), 167.7 (IV), 158.0 (IV), 145.3 (IV), 134.9 (IV), 134.0 (IV), 130.1, 130.0 (2C), 128.8 (2C), 121.0, 116.1, 114.9, 82.3 (IV), 80.5 (IV), 66.6 (2C), 60.2, 59.0, 56.1, 51.9 (2C), 47.3, 38.8, 36.3 and 21.7; HRMS m/z 541.6257 (M $\mathrm{C}_{27} \mathrm{H}_{31} \mathrm{~N}_{3} \mathrm{O}_{7} \mathrm{~S}$ requires 541.6250); Anal. Calcd for $\mathrm{C}_{27} \mathrm{H}_{31} \mathrm{~N}_{3} \mathrm{O}_{7} \mathrm{~S}$ : C, 59.88; H, 5.77; N, 7.76. Found: C, 60.11; H, 5.94; N, 7.70. 
$\mathrm{N}$-Benzyl-2-\{3-[(4-morpholin-4-ylbut-2-ynyl)oxy]phenyl\}-2-(2-oxoazetidin-1-yl)acetamide

(3.6). Crude residue was purified via column chromatography, eluting with $\mathrm{MeOH} / \mathrm{EtOAc}$ (0.3:9.7), to yield the product as a light-brown oil, $(0.168 \mathrm{~g}, 99 \%) ; \mathrm{R}_{\mathrm{f}}(\mathrm{MeOH} / \mathrm{EtOAc}, 0.3: 9.7)$ 0.26 ; IR (DCM, $\left.v_{\max }, \mathrm{cm}^{-1}\right) 2306 \mathrm{w}, 1745 \mathrm{~s}, 1683 \mathrm{~s}, 1264 \mathrm{~s}$ and $1024 \mathrm{~m} ;{ }^{1} \mathrm{H}$ NMR $\left(\mathrm{CDCl}_{3}, 400 \mathrm{MHz}\right.$, $\left.30{ }^{\circ} \mathrm{C}\right): \delta_{\mathrm{H}} 7.62(\mathrm{~m}, 3 \mathrm{H}), 7.12(\mathrm{~m}, 3 \mathrm{H}), 6.95(\mathrm{~m}, 3 \mathrm{H}), 6.76(\mathrm{br} \mathrm{s}, 1 \mathrm{H}), 5.37(\mathrm{~s}, 1 \mathrm{H}), 4.67(\mathrm{~m}, 2 \mathrm{H})$, $4.43(\mathrm{dd}, J 14.8,6.0 \mathrm{~Hz}, 1 \mathrm{H}), 4.38(\mathrm{dd}, J 14.8,6.0 \mathrm{~Hz}, 1 \mathrm{H}), 3.60(\mathrm{~m}, 5 \mathrm{H}), 3.28(\mathrm{~s}, 2 \mathrm{H}), 3.12(\mathrm{td}, J$ 5.6, 5.6, $2.8 \mathrm{~Hz}, 1 \mathrm{H}), 2.93$ (ddd, $J 14.8,5.6,2.4 \mathrm{~Hz}, 1 \mathrm{H}$ ), 2.82 (ddd, $J 14.8,5.6,2.4 \mathrm{~Hz}, 1 \mathrm{H}$ ), 2.45 (m, 4H); ${ }^{13} \mathrm{C} \mathrm{NMR}\left(\mathrm{CDCl}_{3}, 100 \mathrm{MHz}, 30{ }^{\circ} \mathrm{C}\right): \delta_{\mathrm{c}} 168.4$ (IV), 167.9 (IV), 158.1 (IV), 137.8 (IV), 136.0 (IV), 130.1, 128.7 (2C), 127.7 (2C), 127.5, 121.0, 115.6, 115.0, 82.5 (IV), 80.2 (IV), 66.7 (2C), 59.6, 56.2, 52.0 (2C), 47.3, 43.7, 39.0 and 36.3; HRMS $\mathrm{m} / z$ 447.5351 $\left(\mathrm{M}^{+} \mathrm{C}_{26} \mathrm{H}_{29} \mathrm{~N}_{3} \mathrm{O}_{4}\right.$ requires 447.5340); Anal. Calcd for $\mathrm{C}_{26} \mathrm{H}_{29} \mathrm{~N}_{3} \mathrm{O}_{4}: \mathrm{C}, 69.78 ; \mathrm{H}, 6.53 ; \mathrm{N}, 9.39$. Found: $\mathrm{C}, 69.57 ; \mathrm{H}$, $6.41 ; \mathrm{N}, 9.45$.

$\mathrm{N}$-(1H-1,2,3-Benzotriazol-1-ylmethyl)-2-\{3-[(4-morpholin-4-ylbut-2-ynyl)oxy]phenyl\}-2-(2oxoazetidin-1-yl)acetamide (3.7). Crude residue was purified via column chromatography, eluting with EtOAc to yield the product as a brown powder, $(0.113 \mathrm{~g}, 60 \%) ; \mathrm{mp} 131-134{ }^{\circ} \mathrm{C}$; $\mathrm{R}_{\mathrm{f}}(\mathrm{MeOH} / \mathrm{DCM}, 0.5: 9.5)$ 0.21; IR (DCM, $\left.v_{\max }, \mathrm{cm}^{-1}\right) 2163 \mathrm{~m}, 1743 \mathrm{~s}, 1705 \mathrm{~s}, 1265 \mathrm{~s}$ and $1035 \mathrm{~m} ;{ }^{1} \mathrm{H}$ NMR $\left(\mathrm{CDCl}_{3}, 400 \mathrm{MHz}, 30^{\circ} \mathrm{C}\right): \delta_{\text {н }} 8.15(\mathrm{t}, J 6.4 \mathrm{~Hz}, 1 \mathrm{H}) 7.98(\mathrm{dt}, J 8.4,2.0,2.0 \mathrm{~Hz}, 1 \mathrm{H}), 7.87$ $(\mathrm{dt}, J 8.4,2.0,2.0 \mathrm{~Hz}, 1 \mathrm{H}), 7.48(\mathrm{td}, J 7.6,7.6,1.6 \mathrm{~Hz}, 1 \mathrm{H}), 7.35(\mathrm{td}, J 7.6,7.6,1.6 \mathrm{~Hz}, 1 \mathrm{H}), 7.11$ (t, $J 8.4 \mathrm{~Hz}, 1 \mathrm{H}), 6.84(\mathrm{dd}, J 7.6,1.6 \mathrm{~Hz}, 1 \mathrm{H}), 6.80(\mathrm{t}, J 2.0 \mathrm{~Hz}, 1 \mathrm{H}), 6.70(\mathrm{dd}, J 7.6,1.6 \mathrm{~Hz}, 1 \mathrm{H})$, $6.11(\mathrm{dd}, J 14.0,6.4 \mathrm{~Hz}, 1 \mathrm{H}), 5.99(\mathrm{dd}, J 14.0,6.4 \mathrm{~Hz}, 1 \mathrm{H}), 5.38(\mathrm{~s}, 1 \mathrm{H}), 4.55(\mathrm{t}, J 1.6 \mathrm{~Hz}, 2 \mathrm{H})$, $3.66(\mathrm{~m}, 4 \mathrm{H}), 3.51(\mathrm{td}, J 5.6,2.8 \mathrm{~Hz}, 1 \mathrm{H}), 3.28(\mathrm{t}, J 1.6 \mathrm{~Hz}, 2 \mathrm{H}), 3.04(\mathrm{td}, J 5.6,2.8 \mathrm{~Hz}, 1 \mathrm{H}), 2.91$ (ddd, $J 14.4,5.6,2.8 \mathrm{~Hz}, 1 \mathrm{H}), 2.80(\mathrm{ddd}, J=14.4,5.6,2.8 \mathrm{~Hz}, 1 \mathrm{H}), 2.43(\mathrm{~m}, 4 \mathrm{H}) ;{ }^{13} \mathrm{C}$ NMR $\left(\mathrm{CDCl}_{3}, 100 \mathrm{MHz}, 30{ }^{\circ} \mathrm{C}\right.$ ): $\delta \mathrm{c} 169.5$ (IV), 169.4 (IV), 167.9 (IV), 158.0 (IV), 146.0 (IV), 135.0 (IV), 130.1, 128.0, 124.0, 120.8, 119.7, 116.1, 114.9, 110.7, 82.5 (IV), 80.2 (IV), 66.8 (2C), 59.2, 56.0, 51.8 (2C), 51.0, 47.3, 38.8 and 36.4; HRMS $m / z 488.5468\left(\mathrm{M}^{+} \mathrm{C}_{26} \mathrm{H}_{28} \mathrm{~N}_{6} \mathrm{O}_{4}\right.$ requires 488.5461); Anal. Calcd for $\mathrm{C}_{26} \mathrm{H}_{28} \mathrm{~N}_{6} \mathrm{O}_{4}: \mathrm{C}, 63.92 ; \mathrm{H}, 5.78 ; \mathrm{N}, 17.20$. Found: $\mathrm{C}, 64.28$.; H, 6.05; $\mathrm{N}, 17.29$.

\section{Acknowledgements}

The authors acknowledge the Claude Leon Foundation for funding (MB).

\section{References}

1. World Health Organisation, 2014; Vol. 2014. http://www.who.int/mediacentre/factsheets/fs094/en/

2. Ashley, E. A.; Dhorda, M.; Fairhurst, R. M.; Amaratunga, C.; Lim, P.; Suon, S.; Sreng, S.; Anderson, J. M.; Mao, S.; Sam, B. New Engl. J. Med. 2014, 371, 411. 
http://dx.doi.org/10.1056/NEJMoa1314981

3. Domling, A.; Wang, W.; Wang, K. Chem. Rev. 2012, 112, 3083.

http://dx.doi.org/10.1021/cr100233r

4. Domling, A. Chem. Rev. 2006, 106, 17.

http://dx.doi.org/10.1021/cr0505728

5. Sheehan, J. C.; Henery-Logan, K.; Johnson, D. J. Am. Chem. Soc. 1953, 75, 3292. http://dx.doi.org/10.1021/ja01109a526

6. Lewis, K. Nat. Rev. Drug Discov. 2013, 12, 371.

http://dx.doi.org/10.1038/nrd3975

7. Neyer, G.; Achatz, J.; Danzer, B.; Ugi, I. Heterocycles 1990, 30, 863.

http://dx.doi.org/10.3987/COM-89-S68

8. Pirrung, M. C.; Das Sarma, K. J. Am. Chem. Soc. 2004, 126, 444.

http://dx.doi.org/10.1021/ja038583a

9. Pitlik, J.; Townsend, C. A. Bioorg. Med. Chem. Lett. 1997, 7, 3129.

http://dx.doi.org/10.1016/S0960-894X(97)10170-6

10. Trager, W.; Jensen, J. B. Science 1976, 193, 673. http://dx.doi.org/10.1126/science.781840

11. Makler, M. T.; Hinrichs, D. J. Am. J. Trop. Med. Hyg. 1993, 38, 205.

12. Pal, M.; Parasuraman, K.; Yeleswarapu, K. R. Org. Lett. 2003, 5, 349.

http://dx.doi.org/10.1021/o1027382t 